UPR/0852-T CTP TAMU-31/99 SISSA-Ref. 88/99/EP Imperial/TP/98-99/63

NSF-ITP-99/086 July $1999 \quad$ hep-th/9907202

\title{
T-Duality in the Green-Schwarz Formalism, and the Massless/Massive IIA Duality Map
}

\author{
M. Cvetič ${ }^{\dagger+1}$, H. Lü ${ }^{\dagger 1}$, C.N. Pope ${ }^{\ddagger 2}$ and K.S. Stelle ${ }^{\star 3}$ \\ ${ }^{\dagger}$ Dept. of Phys. and Astro., University of Pennsylvania, Philadelphia, PA 19104 \\ + Institute for Theor. Phys., Univ. of Santa Barbara, Santa Barbara, CA 93106 \\ $\ddagger$ Center for Theoretical Physics, Texas A $\mathcal{G} M$ University, College Station, TX 77843 \\ $\ddagger$ SISSA, Via Beirut No. 2-4, 34013 Trieste, Italy \\ ${ }^{\star}$ The Blackett Laboratory, Imperial College, Prince Consort Road, London SW7 $2 B Z$.
}

\begin{abstract}
$\underline{\text { ABSTRACT }}$
We derive a component-field expansion of the Green-Schwarz action for the type IIA string, in an arbitrary background of massless NS-NS and R-R bosonic fields, up to quadratic order in the fermionic coordinates $\theta$. Using this action, we extend the usual derivation of Buscher T-duality rules to include not only NS-NS, but also R-R fields. Our implementation of the T-duality transformation rules makes use of adapted background-field parametrizations, which provide a more geometrically natural and elegant description for the duality maps than the ones previously presented. These T-duality rules allow us to derive the Green-Schwarz action for the type IIB string in an arbitrary background of massless NS-NS and R-R bosonic fields, up to $O\left(\theta^{2}\right)$. Implemention of another T-duality transformation on this type IIB action then allows us also to derive the Green-Schwarz action for the massive IIA string. By further considering T-duality transformations for backgrounds with the two $U(1)$ isometries of a 2-torus, we give a string-theoretic derivation of the direct T-duality relation between the massless and massive type IIA strings. In addition, we give an explicit construction of the $D=8 S L(3, \mathbb{R}) \times S L(2, \mathbb{R})$ invariant supergravity with two mass parameters that form a doublet under the $S L(2, \mathbb{R})$ factor.
\end{abstract}

\footnotetext{
${ }^{1}$ Research supported in part by DOE grant DOE-FG02-95ER40893

${ }^{2}$ Research supported in part by DOE grant DE-FG03-95ER40917

${ }^{3}$ Research supported in part by the EC under TMR contract ERBFMRX-CT96-0045.
} 


\section{Contents}

\begin{tabular}{lll}
\hline 1 & Introduction & 2
\end{tabular}

\begin{tabular}{|lll}
\hline 2 & Type IIA Superstring Action from the Supermembrand & 4
\end{tabular}

\begin{tabular}{|lll|}
\hline 3 & T-duality and the Type IIB Superstring Action & 7
\end{tabular}

3.1 T-duality in the Green-Schwarz action . . . . . . . . . . . . . . . . . . 7

3.2 Type IIA/IIB T-duality $\ldots \ldots \ldots \ldots \ldots$. . . . . . . . . . . . . . . . . . 11

\begin{tabular}{|lll}
\hline & Green-Schwarz Action for the Massive Type IIA Theory & 15
\end{tabular}

$\begin{array}{lll}5 & \text { Massless/massive type IIA T-duality } & 17\end{array}$

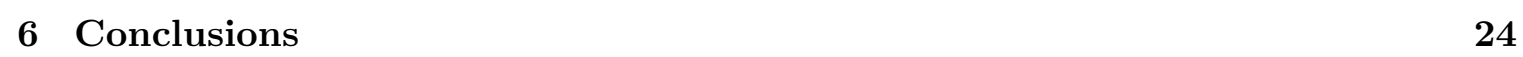

\begin{tabular}{lll}
\hline 7 Addendum & 25
\end{tabular}

\begin{tabular}{|ll}
\hline A Massless/massive IIA T-duality from $D=8$ massive supergravity & $\mathbf{2 7}$
\end{tabular} 


\section{Introduction}

T-duality is the most securely founded of the duality symmetries of string theory, being grounded in worldsheet field manipulations that do not change the corresponding conformal field theory (see reviews [1, 2]). It has been discussed mainly in the Neveu-Schwarz-Ramond formalism, where couplings to the Ramond-Ramond sector of superstring theory do not appear in the classical string action. However, when one is concerned with the effects of T-duality on nontrivial R-R backgrounds, it is more appropriate to use the Green-Schwarz formulation. In this paper we develop procedures for deriving T-duality in the GreenSchwarz formalism. We first apply the method to a derivation of the standard type IIA/IIB string T-duality, including R-R backgrounds. Next, we extend the discussion to allow for backgrounds associated with the massive type IIA theory. Since this extension of the theory inherently involves R-R backgrounds, the Green-Schwarz formalism is ideally suited to describing the extended T-duality symmetries that relate the massive IIA string to the type IIB string. Finally, we apply our discussion of T-duality in the Green-Schwarz formalism to the case of type IIA strings propagating in backgrounds with two abelian isometries. By this means we are able to exhibit explicitly the T-duality relation between the massless and massive IIA theories.

We begin by obtaining a component-field expansion of the superfield form of the GreenSchwarz action for the type IIA superstring in an arbitrary background of bosonic NSNS and R-R fields, working to order $O\left(\theta^{2}\right)$ in the fermionic coordinates. We do this by making a double dimensional reduction [3] starting from the superspace form of the $D=11$ supermembrane action 沺, and using previous results for the component expansions of the $D=11$ superfields [5, 6, 7]. Thus we obtain the component-field expansion of the type IIA Green-Schwarz string action given in (2.8), to $O\left(\theta^{2}\right)$ in the fermionic coordinates, in arbitrary massless bosonic NS-NS and R-R fields.

Performing next the T-duality transformation for a single $U(1)$ isometry, we show how an appropriate set of background-field definitions significantly simplifies the T-duality derived transformations of these fields. We do this by adopting variable choices for the background fields that are geometrically adapted to the background with its assumed abelian isometry, which is necessary for implementation of the T-duality map. This leads to a simple set of expressions for the background-field transformations that precisely matches the known forms of nonlinear symmetry transformations in the corresponding effective supergravity theory. Since, in our discussion, we are using a formalism where all the massless bosonic fields are non-vanishing, this allows us to derive T-duality transformation rules for the 
R-R as well as the NS-NS backgrounds, in a manner directly comparable with previous field-theoretic derivations, but now instead within the framework of the string action.

String-theory T-duality transformations have an advantage over those derived in the effective field theory, in that they are carried out without suppressing string excitations along the directions of the assumed isometries of the background fields. Thus, whereas in supergravity field theories the assumption of isometries means that one has effectively made a Kaluza-Klein reduction to a lower-dimensional theory, this is not the case in string theory. Accordingly, in string theory, one may access the larger groups of nonlinear symmetry transformations that appear in supergravity theories upon dimensional reduction, but without actually sacrificing the higher-dimensional modes of the string itself. Thus in our approach we obtain the type IIA/IIB T-duality in the more general string-theoretic framework, including R-R background fields, while at the same time employing the more geometrically adapted description commonly used in field-theoretic discussions.

The component-field expansion of the usual type IIA Green-Schwarz string that we obtain includes non-vanishing backgrounds for the 2-form and 4-form R-R field strengths $F_{(2)}$ and $F_{(4)}$. By performing a T-duality transformation with a single $U(1)$ isometry, we show how the action can again be interpreted as a covariant ten-dimensional action, but now describing the type IIB string. By this means we derive for the first time a componentfield expansion of the type IIB Green-Schwarz string action, again to $O\left(\theta^{2}\right)$ in the fermionic coordinates, in arbitrary massless bosonic backgrounds, including the 1-form, 3-form and 5-form R-R field strengths $\widetilde{F}_{(1)}=d \chi, \widetilde{F}_{(3)}$ and $\widetilde{F}_{(5)}$. The action is given by (3.28).

From the type IIB Green-Schwarz action we then perform a further T-duality transformation, for a single $U(1)$ isometry along a coordinate $y$, in which the field strength $\widetilde{F}_{(1)}$ is allowed to have a non-vanishing component in the direction of the isometry, $\widetilde{F}_{(1)} \longrightarrow$ $\widetilde{F}_{(1)}+m d y$. From a field-theoretic standpoint this corresponds to performing a ScherkSchwarz generalised reduction, where the axion $\chi$ is allowed a linear dependence on the compactification coordinate $y$. As is well known, this leads to a massive supergravity in $D=9$ which is identical, after a field transformation, to the ordinary dimensional reduction of massive [9] type IIA supergravity [10] (see also [11, 12]). In our discussion of T-duality at the level of the superstring action, we are able to derive the additional 0 -form contribution of the mass term $m$ to the background of the IIA theory, thereby providing an explicit expression for the Green-Schwarz action for the massive IIA string, given by (4.4).

Since the type IIB and the massive type IIA theories are related by an appropriatelygeneralised T-duality on a circle, and on the other hand the massless IIA theory is related 
to the IIB theory by a standard T-duality on a circle, one can expect that it should be possible to derive a direct T-duality relation between the massless and massive IIA theories themselves, in which the background fields are assumed to have the two $U(1)$ isometries of a 2-torus. Indeed, in [13] a massive supergravity was obtained in $D=8$, by performing a generalised Scherk-Schwarz reduction of massless type IIA supergravity. Although not manifestly the same as the theory that one obtains by reducing massive type IIA supergravity on the 2-torus, it is in fact equivalent up to field redefinitions in $D=8$. This formed an ingredient in the discussion in [14], where the notion of massless/massive IIA duality was developed. The arguments presented there involved the comparison of D8-brane and D6-brane solutions of the supergravities in $D=8$. In the present paper, we are able to present a direct and explicit formulation of massless/massive IIA duality, at the level of the Green-Schwarz string action, showing precisely how the T-duality mapping between the two theories is implemented.

\section{Type IIA Superstring Action from the Supermembrane}

We start from the worldvolume action for the supermembrane in $D=11$. This is given by 44

$$
I_{3}=\int d^{3} \xi\left(-\sqrt{-\hat{h}}-\frac{1}{6} \epsilon^{\hat{i} \hat{j} \hat{k}} \partial_{\hat{i}} Z^{M} \partial_{\hat{j}} Z^{N} \partial_{\hat{k}} Z^{P} \hat{A}_{P N M}\right),
$$

where $\hat{h}_{\hat{i} \hat{j}}=\partial_{\hat{i}} Z^{M} \hat{E}_{M}^{\hat{m}} \partial_{\hat{j}} Z^{N} \hat{E}_{N}^{\hat{n}} \eta_{\hat{m} \hat{n}}$ is the world-volume metric, $Z^{M}=\left(X^{\hat{\mu}}, \theta^{\alpha}\right)$ denotes the eleven bosonic and 32 fermionic spacetime coordinates, and $\hat{A}_{P N M}$ is the 3 -form superfield. The supervielbein $\hat{E}_{M}^{A}$ and 3 -form $\hat{A}$ were obtained to leading order in $\theta$ in [5, 6], and more recently they were completely calculated to $O\left(\theta^{2}\right)[7]$. To this order, setting the spinor background fields to zero, they are given by:

$$
\begin{aligned}
& \hat{E}_{\hat{\mu}}^{\hat{m}}=\hat{e}_{\hat{\mu}}^{\hat{m}}+\mathrm{i} \bar{\theta} \Gamma^{\hat{m}}\left(\frac{1}{4} \hat{\omega}_{\hat{\mu}}^{\hat{n} \hat{p}} \Gamma^{\hat{n} \hat{p}}+\hat{T}_{\hat{\mu}}^{\hat{n} \hat{p} \hat{q} \hat{r}} \hat{F}_{\hat{n} \hat{p} \hat{q} \hat{r}) \theta}\right. \\
& \hat{E}_{\hat{\mu}}^{a}=\frac{1}{4} \hat{\omega}_{\hat{\mu}}^{\hat{m} \hat{n}}\left(\Gamma_{\hat{m} \hat{n}} \theta\right)^{a}+\left(\hat{T}_{\hat{\mu}}^{\hat{m} \hat{n} \hat{p} \hat{q}} \theta\right)^{a} \hat{F}_{\hat{m} \hat{n} \hat{p} \hat{q}}, \\
& \hat{E}_{\alpha}^{\hat{m}}=-\mathrm{i}\left(\bar{\theta} \Gamma^{\hat{m}}\right)_{\alpha}, \quad \hat{E}_{\alpha}^{a}=\delta_{\alpha}^{a}+M_{\alpha}^{a}, \\
& \hat{A}_{\hat{\mu} \hat{\nu} \hat{\rho}}=A_{\hat{\mu} \hat{\nu} \hat{\rho}}-3 \mathrm{i} \bar{\theta} \hat{\Gamma}_{[\hat{\mu} \hat{\nu}}\left(\frac{1}{4} \hat{\omega}_{\hat{\rho}]}^{\hat{m} \hat{n}} \Gamma_{\hat{m} \hat{n}}+\hat{T}_{\hat{\rho}]}^{\hat{m} \hat{n} \hat{p} \hat{q}} \hat{F}_{\hat{m} \hat{n} \hat{p} \hat{q}}\right) \theta \text {, } \\
& \left.\hat{A}_{\hat{\mu} \hat{\nu} \alpha}=\mathrm{i}\left(\bar{\theta} \hat{\Gamma}_{\hat{\mu} \hat{\nu}}\right)_{\alpha}, \quad \hat{A}_{\hat{\mu} \alpha \beta}=-\left(\bar{\theta} \hat{\Gamma}_{\hat{\mu} \hat{\nu}}\right)_{(\alpha}\left(\bar{\theta} \hat{\Gamma}^{\hat{\nu}}\right)_{\beta}\right), \\
& \hat{A}_{\alpha \beta \gamma}=-\mathrm{i}\left(\bar{\theta} \hat{\Gamma}_{\hat{\mu} \hat{\nu}}\right)_{(\alpha}\left(\bar{\theta} \hat{\Gamma}^{\hat{\mu}}\right)_{\beta}\left(\bar{\theta} \hat{\Gamma}^{\hat{\nu}}\right)_{\gamma)} \cdot
\end{aligned}
$$

The notation here is as follows. Hatted indices $\hat{\mu}$ are used for eleven-dimensional bosonic coordinates and hatted indices $\hat{m}$ are used for the eleven-dimensional bosonic tangent-space. 
Fermionic coordinate and tangent-space indices are denoted by $\alpha$ and $a$ respectively. The quantity $\hat{T}_{\hat{\mu}}^{\hat{\nu} \hat{\rho} \hat{\sigma} \hat{\lambda}}$ is given by

$$
\hat{T}_{\hat{\mu}}^{\hat{\nu} \hat{\rho} \hat{\sigma} \hat{\lambda}}=\frac{1}{288}\left(\hat{\Gamma}_{\hat{\mu}}^{\hat{\nu} \hat{\rho} \hat{\sigma} \hat{\lambda}}-8 \delta_{\hat{\mu}}^{[\hat{\nu}} \hat{\Gamma}^{\hat{\rho} \hat{\sigma} \hat{\lambda}]}\right)
$$

Whenever coordinate indices appear on the Dirac matrices, we place hats on the matrices to indicate that the eleven-dimensional bosonic vielbein $\hat{e}_{\hat{\mu}}^{\hat{m}}$ has been used. The quantity $M_{\alpha}^{a}$ appearing in (2.2) is of order $\theta^{2}$, but does not contribute to any results at this order.

We now perform a double-dimensional reduction from $D=11$, in order to obtain the superspace action for the type IIA string in $D=10$, expanded to order $\theta^{2}$, in the presence of bosonic background fields. The bosonic coordinates $X^{\hat{\mu}}$ are split as $X^{\hat{\mu}}=\left(X^{\mu}, y\right)$, and the eleventh spacetime coordinate $y$ is set equal to the third world-volume coordinate $\xi^{3}$; $y=\xi^{3}$. Otherwise, all spacetime coordinates are taken to be independent of $\xi^{3}$, and the background fields are taken to be independent of $y$. The reduction ansätze for these bosonic background fields are the usual ones, namely

$$
\begin{aligned}
d \hat{s}_{11}^{2} & =e^{-\frac{2}{3} \phi} d s_{10}^{2}+e^{\frac{4}{3} \phi}\left(d y+A_{(1)}\right)^{2}, \\
\hat{A}_{(3)} & =A_{(3)}+A_{(2)} \wedge d y,
\end{aligned}
$$

where $d \hat{s}_{11}^{2}=\hat{g}_{\hat{\mu} \hat{\nu}} d X^{\hat{\mu}} d X^{\hat{\nu}}$ and $d s_{10}^{2}=g_{\mu \nu} d X^{\mu} d X^{\nu}$, with the metrics given by $\hat{g}_{\hat{\mu} \hat{\nu}}=$ $\hat{e}_{\hat{\mu}}^{\hat{m}} \hat{e}_{\hat{\nu}}^{\hat{n}} \eta_{\hat{m} \hat{n}}$ and $g_{\mu \nu}=e_{\mu}^{m} e_{\nu}^{n} \eta_{m n}$ respectively. Note that the ten-dimensional metric is in the string frame here. We also choose to make a rescaling of the fermionic coordinates, in the process of reducing from $D=11$ to $D=10$, namely, $\theta \longrightarrow e^{-\frac{1}{6} \phi} \theta$. This ensures that in $D=10$, the supercoordinate transformations $\delta X^{\mu}=\mathrm{i} \bar{\theta} \Gamma^{\mu} \epsilon$ and $\delta \theta=\epsilon$ will take their canonical forms.

In order to derive the ten-dimensional type IIA superstring action from the elevendimensional supermembrane action (2.1) using this reduction scheme, one can follow either of two procedures. One may first obtain the ten-dimensional supervielbein components by dimensional reduction in superspace, and then substitute them into the superspace version of the $D=10$ type IIA superstring action,

$$
I_{2}=\int d^{2} \xi\left(-\sqrt{-h}-\frac{1}{2} \epsilon^{i j} \partial_{\hat{i}} Z^{M} \partial_{\hat{j}} Z^{N} A_{N M}\right)
$$

or alternatively one may start directly from the $D=11$ action (2.1) and work out the reduction directly in the action at the component-field level. In the first procedure, one has

\footnotetext{
${ }^{1}$ Note that we have changed from the spinor-conjugation convention used in [7], where $\bar{\psi}=\mathrm{i} \psi^{\dagger} \Gamma^{t}$, to the more familiar one where $\bar{\psi}=\psi^{\dagger} \Gamma^{t}$. See the Addendum section 7 for a more detailed discussion.
} 
to be careful to apply a local Lorentz transformation in superspace in order to put the $D=$ 11 vielbein into Kaluza-Klein reduction form, with $\hat{E}_{y}^{m}=0$, and one also needs to perform a superspace Weyl rescaling [3, 15] $\left(\hat{E}_{\mu}^{m}=\Phi^{-\frac{1}{3}} E_{\mu}^{m}, \hat{E}_{\alpha}^{m}=\Phi^{-\frac{1}{3}} e^{\frac{1}{6} \phi} E_{\alpha}^{m}\right)$, where $\Phi^{\frac{2}{3}}=\hat{E}_{y}^{11}$. It is also convenient to perform additional ten-dimensional local Lorentz transformations to simplify the result. For the vielbein components needed in the type IIA string action, we then obtain after some algebra the following

$$
\begin{aligned}
E_{\mu}^{m}= & e_{\mu}^{m}+\frac{\mathrm{i}}{4} \omega_{\mu}^{p q} \bar{\theta} \Gamma^{m} \Gamma_{p q} \theta-\frac{\mathrm{i}}{8} \bar{\theta} \Gamma_{11} \Gamma^{m} \Gamma^{p q} \theta F_{\mu p q}+\frac{\mathrm{i}}{16} e^{\phi} \bar{\theta} \Gamma_{11} \Gamma^{m} \Gamma^{p q} \Gamma_{\mu} \theta F_{p q} \\
& +\frac{\mathrm{i}}{192} e^{\phi} \bar{\theta} \Gamma^{m} \Gamma^{p_{1} \cdots p_{4}} \Gamma_{\mu} \theta F_{p_{1} \cdots p_{4}}, \\
E_{\alpha}^{m}= & -\mathrm{i}\left(\bar{\theta} \Gamma^{m}\right)_{\alpha} .
\end{aligned}
$$

The type IIA superstring action may then be obtained by inserting these expressions, together with the corresponding reduction of the 3-form field $A$, into the $D=10$ superspace form (2.6) of the string action. Alternatively, one may apply the reduction scheme directly in the eleven-dimensional action (2.1). In either way we find, after some lengthy algebra, that the ten-dimensional type IIA string action, for arbitrary bosonic background fields and expanded to $O\left(\theta^{2}\right)$, is given by $I_{2}=\int d^{2} \xi \mathcal{L}_{2}$, wheref

$$
\begin{aligned}
\mathcal{L}_{2}= & -\frac{1}{2} \sqrt{-h} h^{i j} \partial_{i} X^{\mu} \partial_{j} X^{\nu} g_{\mu \nu}+\frac{1}{2} \epsilon^{i j} \partial_{i} X^{\mu} \partial_{j} X^{\nu} A_{\mu \nu} \\
& -\mathrm{i} \bar{\theta}\left(\sqrt{-h} h^{i j}-\epsilon^{i j} \Gamma_{11}\right) \Gamma_{\mu} D_{j} \theta \partial_{i} X^{\mu} \\
& +\frac{i}{8} \partial_{i} X^{\mu} \partial_{j} X^{\nu} \bar{\theta}\left(\sqrt{-h} h^{i j}-\epsilon^{i j} \Gamma_{11}\right) \Gamma_{11} \Gamma_{\mu}{ }^{\rho \sigma} \theta F_{\nu \rho \sigma} \\
& -\frac{\mathrm{i}}{16} \partial_{i} X^{\mu} \partial_{j} X^{\nu} e^{\phi} \bar{\theta}\left(\sqrt{-h} h^{i j}-\epsilon^{i j} \Gamma_{11}\right)\left(\Gamma_{11} \Gamma_{\mu} \Gamma^{\rho \sigma} \Gamma_{\nu} F_{\rho \sigma}+\frac{1}{12} \Gamma_{\mu} \Gamma^{\rho \sigma \lambda \tau} \Gamma_{\nu} F_{\rho \sigma \lambda \tau}\right) \theta,
\end{aligned}
$$

where

$$
D_{i} \theta \equiv \partial_{i} \theta+\frac{1}{4} \partial_{i} X^{\mu} \omega_{\mu}^{m n} \Gamma_{m n} \theta
$$

The field strengths are given by

$$
F_{(4)}=d A_{(3)}-A_{(1)} \wedge d A_{(2)}, \quad F_{(3)}=d A_{(2)}, \quad F_{(2)}=d A_{(1)} .
$$

For later convenience, the gamma matrix $\Gamma_{11}$ in $\left.2.7,2.8\right)$ is taken to be the negative of the matrix $\Gamma_{\hat{m}}$ that corresponds to setting $\hat{m}$ equal to its eleventh value. $\Gamma_{11}$ is then the chirality operator in $D=10.]^{3}$

\footnotetext{
${ }^{2} \mathrm{~A} \Gamma_{11}$ factor was accidentally omitted in the $F_{\nu \rho \sigma}$ terms in the earlier and published versions of this paper. See the Addendum section 7 for a detailed discussion of corrections and changes of convention and notation.

${ }^{3}$ Our conventions are as follows. The Dirac matrices satisfy $\left\{\Gamma_{\hat{m}}, \Gamma_{\hat{n}}\right\}=2 \eta_{\hat{m} \hat{n}}$, with $\eta=\operatorname{diag}(-1,+1, \ldots+$ 1). For a Majorana spinor $\theta$, the expressions $\bar{\theta} \Gamma_{\hat{m}_{1} \cdots \hat{m}_{p}} \theta$ vanish for $p=(1,2,5,6,9,10)$ and are non-vanishing for $p=(0,3,4,7,8,11)$.
} 
A number of remarks on this result are now in order. If we consider a background in which only the metric and 2 -form potential $A_{\mu \nu}$ are excited, the top two lines in (2.8) are precisely of the standard form, up to $O\left(\theta^{2}\right)$. The third line describes an additional $O\left(\theta^{2}\right)$ coupling of the NS-NS field strength $F_{(3)}$, over and above the standard bosonic coupling to the worldsheet. The final line in (2.8) describes the couplings of the R-R background fields $F_{(2)}$ and $F_{(4)}$. The factor of $e^{\phi}$ that multiplies the R-R terms in (2.8) implies that $\theta$ loop diagrams involving two insertions of the $\mathrm{R}-\mathrm{R}$ vertices will give rise to the expected additional $e^{2 \phi}$ factor in the couplings of the kinetic terms of the R-R fields in the low-energy effective action.

One further remark that is appropriate at this stage is to note that there is no $\sqrt{-h} R_{(2)} \phi$ coupling of the dilaton to the worldsheet. This is what one should expect; such a term would explicitly break the conformal invariance of the classical world-sheet action (2.8), which would contradict the manifest conformal invariance of the classical reduction procedure (see [15]). Moreover, a $\sqrt{-h} R_{(2)} \phi$ term would also break the manifest spacetime supersymmetry of the Green-Schwarz formalism. Whether such a term is actually needed in this formalism remains an open question. In order to calculate conformal anomalies in the Green-Schwarz formalism, one must carefully fix the $\kappa$-symmetry gauge and handle its infinitely recursive ghost sector. In the explicit $\beta$-function calculations that have been performed, for the Green-Schwarz heterotic string at the one and two-loop levels [16], no need has been found for a $\sqrt{-h} R_{(2)} \phi$ term. It may be that such a term only appears out of the quantum measure upon making the variable changes needed for a transition from the Neveu-Schwarz-Ramond to the Green-Schwarz formalism.

\section{T-duality and the Type IIB Superstring Action}

\subsection{T-duality in the Green-Schwarz action}

T-duality in the NS-NS sector has been extensively studied at the level of the string sigma model, starting with [18]. For the type I string, the resulting "Buscher rules" correspond in $D=9$ to a discrete $Z_{2}$ symmetry. For type IIA and IIB strings, whose NS-NS sectors coincide but whose R-R sectors differ, it corresponds to a transformation that maps back and forth between the two theories. In an NSR type of sigma model, one does not see the couplings of the R-R background fields to the worldsheet, and so the methods used in 18]

\footnotetext{
${ }^{4}$ The odd-parity R-R terms proportional to $\epsilon^{i j}$ were presented in [17]; they are in broad structural agreement with ours.
} 
are not directly applicable to the complete type II theories. In fact until now, the analogues of the Buscher rules for the R-R fields have been derived only at the level of the effective low-energy field theory, by performing standard Kaluza-Klein reductions of the type IIA and IIB supergravities and making the necessary identifications of fields that relate the two theories in $D=9$, as in $[8,10]$. In this section, we shall derive the generalisation of the Buscher rules for the Green-Schwarz superstring actions. Since, as we have presented in the previous section, these actions include the couplings of the R-R fields, this will allow us to obtain a worldsheet derivation of the "Buscher rules" for the R-R fields.

To begin, we write down a generic worldsheet Lagrangian with the general structure of (2.8):

$$
\begin{aligned}
\mathcal{L}= & -\frac{1}{2} \sqrt{-h} h^{i j} \partial_{i} X^{\mu} \partial_{j} X^{\nu} G_{\mu \nu}+\frac{1}{2} \epsilon^{i j} \partial_{i} X^{\mu} \partial_{j} X^{\nu} B_{\mu \nu} \\
& +\sqrt{-h} h^{i j} \partial_{i} X^{\mu} \mathcal{G}_{j \mu}+\epsilon^{i j} \partial_{i} X^{\mu} \mathcal{B}_{j \mu} .
\end{aligned}
$$

Note that by comparing this general form with (2.8), the quantities $G_{\mu \nu}$ and $B_{\mu \nu}$ will contain not only the usual $\theta$-independent terms $g_{\mu \nu}$ and $A_{\mu \nu}$, but also certain $\theta^{2}$ terms as well. In fact, it will prove useful to write these as

$$
G_{\mu \nu}=\mathbf{g}_{\mu \nu}+Q_{\mu \nu}, \quad B_{\mu \nu}=A_{\mu \nu}+P_{\mu \nu},
$$

where $Q_{\mu \nu}=Q_{(\mu \nu)}$ and $P_{\mu \nu}=P_{[\mu \nu]}$ represent the symmetric and antisymmetric $O\left(\theta^{2}\right)$ terms coupling to $\partial_{i} X^{\mu} \partial_{j} X^{\nu}$. The quantities $\mathcal{G}_{j \mu}$ and $\mathcal{B}_{j \mu}$, which couple to the single $\partial_{i} X^{\mu}$ pullback, will correspond to the $\bar{\theta} \partial_{i} \theta$ parts of the fermionic kinetic terms in (2.8).

We now follow the analogue of the standard procedure developed for the bosonic string in [18]. Thus we suppose that there is an abelian isometry of the ten-dimensional background fields, such that in an adapted coordinate system the fields are all independent of the tenth of the coordinates $X^{\mu}$. We shall accordingly decompose the coordinates as $X^{\mu}=\left(X^{\mu^{\prime}}, Y\right)$. The next step is to replace $\partial_{i} Y$ in the Lagrangian (3.1) by a worldsheet vector $V_{i}$, at the same time introducing a Lagrange multiplier $Z$ (a worldsheet scalar) and adding the term $\epsilon^{i j} \partial_{i} Z V_{j}$. The equation of motion for $Z$ enforces the irrotationality of $V_{i}$. By instead varying the action with respect to $V_{i}$, and substituting its algebraic solution back into the action, we obtain

$$
\begin{aligned}
\mathcal{L}= & -\frac{1}{2} \sqrt{-h} h^{i j} \partial_{i} X^{\mu^{\prime}} \partial_{j} X^{\nu^{\prime}} G_{\mu^{\prime} \nu^{\prime}}+\frac{1}{2} \epsilon^{i j} \partial_{i} X^{\mu^{\prime}} \partial_{j} X^{\nu^{\prime}} B_{\mu^{\prime} \nu^{\prime}} \\
& +\sqrt{-h} h^{i j} \partial_{i} X^{\mu^{\prime}} \mathcal{G}_{j \mu^{\prime}}+\epsilon^{i j} \partial_{i} X^{\mu^{\prime}} \mathcal{B}_{j \mu^{\prime}} \\
& -\frac{1}{2} \sqrt{-h} h^{i j} G_{00}^{-1}\left(u_{i} u_{j}-v_{i} v_{j}\right)+\epsilon^{i j} G_{00}^{-1} u_{i} v_{j},
\end{aligned}
$$


where

$$
u_{i} \equiv \partial_{i} Z+\partial_{i} X^{\mu^{\prime}} B_{\mu^{\prime} 0}-\mathcal{B}_{i 0}, \quad v_{i} \equiv-\partial_{i} X^{\mu^{\prime}} G_{\mu^{\prime} 0}+\mathcal{G}_{i 0}
$$

Note that, following the conventional notation, we associate the index " 0 " with the $Y$ coordinate.

The Lagrangian (3.3) can be seen to be of the same general form as (3.1), with $Z$ rather than $Y$ now viewed as the tenth coordinate. Recasting (3.3) in the form (3.1) implies that there will now be a transformed set of background fields, which we shall denote by $\widetilde{G}_{\mu \nu}$, $\widetilde{B}_{\mu \nu}, \widetilde{\mathcal{G}}_{j \mu}$ and $\widetilde{\mathcal{B}}_{j \mu}$. Recalling that $G_{\mu \nu}$ and $B_{\mu \nu}$ include terms both of order $\theta^{0}$ and $\theta^{2}$, as indicated in (3.2), we may then read off the full set of transformed background fields, correct to order $\theta^{2}$. At zeroth order in $\theta$ we find the usual Buscher rules,

$$
\begin{aligned}
\tilde{\mathbf{g}}_{\mu^{\prime} \nu^{\prime}} & =\mathbf{g}_{\mu^{\prime} \nu^{\prime}}+\mathbf{g}_{00}^{-1}\left(A_{\mu^{\prime} 0} A_{\nu^{\prime} 0}-\mathbf{g}_{\mu^{\prime} 0} \mathbf{g}_{\nu^{\prime} 0}\right) \\
\tilde{\mathbf{g}}_{\mu^{\prime} 0} & =\mathbf{g}_{00}^{-1} A_{\mu^{\prime} 0}, \quad \tilde{\mathbf{g}}_{00}=\mathbf{g}_{00}^{-1}, \\
\widetilde{A}_{\mu^{\prime} \nu^{\prime}} & =A_{\mu^{\prime} \nu^{\prime}}+\mathbf{g}_{00}^{-1}\left(\mathbf{g}_{\mu^{\prime} 0} A_{\nu^{\prime} 0}-\mathbf{g}_{\nu^{\prime} 0} A_{\mu^{\prime} 0}\right) \\
\widetilde{A}_{\mu^{\prime} 0} & =\mathbf{g}_{00}^{-1} \mathbf{g}_{\mu^{\prime} 0} .
\end{aligned}
$$

At $O\left(\theta^{2}\right)$, we obtain the following rules:

$$
\begin{aligned}
\widetilde{Q}_{\mu^{\prime} \nu^{\prime}} & =Q_{\mu^{\prime} \nu^{\prime}}+2 \mathbf{g}_{00}^{-1}\left(P_{\left(\mu^{\prime}|0|\right.} A_{\left.\nu^{\prime}\right) 0}-Q_{\left(\mu^{\prime}|0|\right.} \mathbf{g}_{\left.\nu^{\prime}\right) 0}\right)+\mathbf{g}_{00}^{-2}\left(\mathbf{g}_{\mu^{\prime} 0} \mathbf{g}_{\nu^{\prime} 0}-A_{\mu^{\prime} 0} A_{\nu^{\prime} 0}\right) Q_{00} \\
\widetilde{Q}_{\mu^{\prime} 0} & =\mathbf{g}_{00}^{-1} P_{\mu^{\prime} 0}-\mathbf{g}_{00}^{-2} A_{\mu^{\prime} 0} Q_{00}, \quad \widetilde{Q}_{00}=-\mathbf{g}_{00}^{-2} Q_{00} \\
\widetilde{P}_{\mu^{\prime} \nu^{\prime}} & =P_{\mu^{\prime} \nu^{\prime}}-2 \mathbf{g}_{00}^{-1}\left(P_{\left[\mu^{\prime}|0|\right.} \mathbf{g}_{\left.\nu^{\prime}\right] 0}-Q_{\left[\mu^{\prime}|0|\right.} A_{\left.\nu^{\prime}\right] 0}\right)+2 \mathbf{g}_{00}^{-2} Q_{00} A_{\left[\mu^{\prime}|0|\right.} \mathbf{g}_{\left.\nu^{\prime}\right] 0} \\
\widetilde{P}_{\mu^{\prime} 0} & =\mathbf{g}_{00}^{-1} Q_{\mu^{\prime} 0}-\mathbf{g}_{00}^{-2} \mathbf{g}_{\mu^{\prime} 0} Q_{00}, \\
\widetilde{\mathcal{G}}_{i \mu^{\prime}} & =\mathcal{G}_{i \mu^{\prime}}+\mathbf{g}_{00}^{-1}\left(\mathcal{B}_{i 0} A_{\mu^{\prime} 0}-\mathcal{G}_{i 0} \mathbf{g}_{\mu^{\prime} 0}\right), \quad \widetilde{\mathcal{G}}_{i 0}=\mathbf{g}_{00}^{-1} \mathcal{B}_{i 0} \\
\widetilde{\mathcal{B}}_{i \mu^{\prime}} & =\mathcal{B}_{i \mu^{\prime}}+\mathbf{g}_{00}^{-1}\left(\mathcal{G}_{i 0} A_{\mu^{\prime} 0}-\mathcal{B}_{i 0} \mathbf{g}_{\mu^{\prime} 0}\right), \quad \widetilde{\mathcal{B}}_{i 0}=\mathbf{g}_{00}^{-1} \mathcal{G}_{i 0}
\end{aligned}
$$

In these formulae we have followed tradition [18, in denoting the components of the metric (the $\theta$-independent terms in $G_{\mu^{\prime} \nu^{\prime}}, G_{\mu^{\prime} 0}$ and $G_{00}$ ) simply as $\mathbf{g}_{\mu^{\prime} \nu^{\prime}}, \mathbf{g}_{\mu^{\prime} 0}$ and $\mathbf{g}_{00}$. There is, however, a much more natural way to parametrize the ten-dimensional metric in terms of nine-dimensional fields, namely by using the standard Kaluza-Klein decomposition from $D=10$ to $D=9$. Thus the natural metric to consider in $D=9$ is not $\mathbf{g}_{\mu^{\prime} \nu^{\prime}}$, but rather, $g_{\mu^{\prime} \nu^{\prime}}=\mathbf{g}_{\mu^{\prime} \nu^{\prime}}+\mathbf{g}_{00}^{-1} \mathbf{g}_{\mu^{\prime} 0} \mathbf{g}_{\nu^{\prime} 0}$. (For the mixed and the internal components, there is no need to make a distinction between $\mathbf{g}$ and $g$.) This can be seen easily from the form of the string-frame Kaluza-Klein reduction ansatz, which is

$$
d \hat{s}^{2}=d s^{2}+e^{2 \lambda}\left(d z+\mathcal{A}_{(1)}\right)^{2},
$$


where $d s^{2}=g_{\mu^{\prime} \nu^{\prime}} d X^{\mu^{\prime}} d X^{\nu^{\prime}}$, and $\mathcal{A}_{(1)}=\mathcal{A}_{\mu^{\prime}} d X^{\mu^{\prime}}$ is the Kaluza-Klein vector. In the type IIA theory, we have $\lambda=\frac{1}{4} \phi+\frac{\sqrt{7}}{4} \varphi$. In terms of the metric decomposition (3.7), it is easy to see that the standard bosonic Buscher rules (3.5) can be rewritten in the more elegant form

$$
\begin{aligned}
\tilde{g}_{\mu^{\prime} \nu^{\prime}}=g_{\mu^{\prime} \nu^{\prime}}, \quad \widetilde{\mathcal{A}}_{(1)}=A_{(1)}, & \tilde{\lambda}=-\lambda, \\
\widetilde{A}_{(2)}= & A_{(2)}+\mathcal{A}_{(1)} \wedge A_{(1)}, \quad \widetilde{A}_{(1)}=\mathcal{A}_{(1)},
\end{aligned}
$$

where the 2 -form potential in $D=10$ is reduced to $D=9$ according to the standard KaluzaKlein prescription $A_{(2)} \longrightarrow A_{(2)}+A_{(1)} \wedge d z$. Note that the expressions in (3.8) are identical to those that one finds at the field-theory level, when mapping the dimensionally-reduced type IIA and IIB supergravities into one another in $D=9$. (See, for example, [12].)

For the $O\left(\theta^{2}\right)$ terms (3.6), we can also obtain a more elegant form by using the technique, familiar in Kaluza-Klein reductions, of using tangent-space rather than coordinate indices. Upon doing so, we find that (3.6) can be re-expressed in the considerably simpler form

$$
\begin{array}{rlrl}
\widetilde{Q}_{m^{\prime} n^{\prime}} & =Q_{m^{\prime} n^{\prime}}, \quad \widetilde{Q}_{m^{\prime} 0}=P_{m^{\prime} 0}, \quad \widetilde{Q}_{00}=-Q_{00}, \\
\widetilde{P}_{m^{\prime} n^{\prime}} & =P_{m^{\prime} n^{\prime}}, \quad \widetilde{P}_{m^{\prime} 0}=Q_{m^{\prime} 0}, & \\
\widetilde{\mathcal{G}}_{i m^{\prime}}=\mathcal{G}_{i m^{\prime}}, & \widetilde{\mathcal{G}}_{i 0}=\mathcal{B}_{i 0}, \\
\widetilde{\mathcal{B}}_{i m^{\prime}}=\mathcal{B}_{i m^{\prime}}, & \widetilde{\mathcal{B}}_{i 0}=\mathcal{G}_{i 0},
\end{array}
$$

where here, $m^{\prime}$ and 0 now denote tangent-space indices. Henceforth, we shall always use tangent-space indices on $Q_{m n}$ and $P_{m n}$. Note that all the type IIA and IIB field strengths that appear in our results, including all their non-linear corrections, will now be precisely the same as one finds in the corresponding supergravity theories. In the conventions that we are using in this paper, their precise expressions may all be found in [19].

In order to discuss T-duality in the $O\left(\theta^{2}\right)$ sector, we must make a nine-dimensional decomposition of the spinor coordinates. The spinor $\theta$ in $D=10$ has 32 components, split into 16 chiral and 16 antichiral components. In $D=9$, we therefore obtain two 16-component spinors. We shall decompose the ten-dimensional Dirac matrices as follows:

$$
\Gamma_{m^{\prime}}=\left(\begin{array}{cc}
0 & \gamma_{m^{\prime}} \\
\gamma_{m^{\prime}} & 0
\end{array}\right), \quad \Gamma_{0}=\left(\begin{array}{cc}
0 & \mathrm{i} \\
-\mathrm{i} & 0
\end{array}\right), \quad \Gamma_{11}=\left(\begin{array}{cc}
\mathbb{1} & 0 \\
0 & -\mathbb{1}
\end{array}\right),
$$

where $m^{\prime}$ is a tangent-space index running over the nine-dimensional range, with corresponding $16 \times 16$ Dirac matrices $\gamma_{m^{\prime}}$. It should be emphasised that $\Gamma_{0}$ here refers to the Dirac matrix of the tenth direction (and not the time direction!). The 32-component spinor 
$\theta$ of the type IIA theory (2.8) then takes the form $\theta=\theta^{1}+\theta^{2}$, where

$$
\theta^{1}=\left(\begin{array}{c}
\vartheta^{1} \\
0
\end{array}\right), \quad \theta^{2}=\left(\begin{array}{c}
0 \\
\vartheta^{2}
\end{array}\right) .
$$

Thus we have $\Gamma_{11} \theta^{1}=\theta^{1}$ and $\Gamma_{11} \theta^{2}=-\theta^{2}$.

In the type IIB theory, which we shall obtain by performing a T-duality transformation, there are two Majorana spinors of positive chirality in $D=10$, which we shall denote by $\tilde{\theta}^{A}$, with $A=1,2$. These will be related to the spinors $\theta^{A}$ of the type IIA theory by

$$
\tilde{\theta}^{1}=\theta^{1}, \quad \tilde{\theta}^{2}=\Gamma_{0} \theta^{2} .
$$

\subsection{Type IIA/IIB T-duality}

With these preliminaries, we are now ready to implement the T-duality transformation on the type IIA action (2.8). The new part of our construction, which goes beyond previous results, involves the consideration of the $O\left(\theta^{2}\right)$ terms, and, in particular, the R-R field couplings. Firstly, we read off from (2.8) the various $O\left(\theta^{2}\right)$ terms in (3.1). In order to bring out the parallelism between the type IIA and type IIB theories, we express the $\theta$ coordinates of the IIA theory in the notation $\theta^{A}$ introduced above. Thus for the terms coupling to NS-NS background fields we have

$$
\begin{aligned}
Q_{m n}^{\mathrm{NS}} & =\frac{\mathrm{i}}{4}\left[\bar{\theta}^{1} \Gamma_{(m}{ }^{p q} \theta^{1}-\bar{\theta}^{2} \Gamma_{(m}^{p q} \theta^{2}\right] F_{n) p q}^{\mathrm{NS}}+\frac{\mathrm{i}}{2}\left[\bar{\theta}^{1} \Gamma_{(m} \Gamma_{|p q|} \theta^{1}+\bar{\theta}^{2} \Gamma_{(m} \Gamma_{|p q|} \theta^{2}\right] \omega_{n)}{ }^{p q}, \\
P_{m n}^{\mathrm{NS}} & =-\frac{\mathrm{i}}{4}\left[\bar{\theta}^{1} \Gamma_{(m}{ }^{p q} \theta^{1}+\bar{\theta}^{2} \Gamma_{(m}{ }^{p q} \theta^{2}\right] F_{n) p q}^{\mathrm{NS}}-\frac{\mathrm{i}}{2}\left[\bar{\theta}^{1} \Gamma_{(m} \Gamma_{|p q|} \theta^{1}-\bar{\theta}^{2} \Gamma_{(m} \Gamma_{|p q|} \theta^{2}\right] \omega_{n)}{ }^{p q}, \\
\mathcal{G}_{i m} & =-\mathrm{i}\left[\bar{\theta}^{1} \Gamma_{m} \partial_{i} \theta^{1}+\bar{\theta}^{2} \Gamma_{m} \partial_{i} \theta^{2}\right], \\
\mathcal{B}_{i m} & =-\mathrm{i}\left[\bar{\theta}^{1} \Gamma_{m} \partial_{i} \theta^{1}-\bar{\theta}^{2} \Gamma_{m} \partial_{i} \theta^{2}\right] .
\end{aligned}
$$

(Note that $\omega_{n}^{p q}$ denotes the tangent-space components of the spin connection; i.e. $\omega^{p q}=$ $\omega_{n}^{p q} e^{n}$.) Similarly, we can read off from (2.8) the contributions to $Q_{m n}$ and $P_{m n}$ coming from the R-R sector:

$$
\begin{aligned}
Q_{m n}^{(2)} & =-\frac{i m}{4} e^{\phi} \bar{\theta}^{[1} \Gamma_{(m} \Gamma^{p q} \Gamma_{n)} \theta^{2]} F_{p q}, \\
P_{m n}^{(2)} & =\frac{\mathrm{i}}{4} e^{\phi} \bar{\theta}^{(1} \Gamma_{[m} \Gamma^{p q} \Gamma_{n]} \theta^{2)} F_{p q}, \\
Q_{m n}^{(4)} & =\frac{\mathrm{i}}{48} e^{\phi} \bar{\theta}^{(1} \Gamma_{(m} \Gamma^{p q r s} \Gamma_{n)} \theta^{2)} F_{p q r s}, \\
P_{m n}^{(4)} & =-\frac{\mathrm{i}}{48} e^{\phi} \bar{\theta}^{[1} \Gamma_{[m} \Gamma^{p q r s} \Gamma_{n]} \theta^{2]} F_{p q r s},
\end{aligned}
$$

\footnotetext{
${ }^{5}$ The definition of $\tilde{\theta}^{2}$ that we are now using differs from that used in the version of this paper published in Nucl. Phys. B573 (2000) 149, and in v1. of hep-th/9907202, where we defined $\tilde{\theta}^{2}=-\mathrm{i} \Gamma_{0} \theta^{2}$. While there was nothing wrong with taking that definition, it did mean that the two chiral spinors $\tilde{\theta}^{1}$ and $\tilde{\theta}^{2}$ in the type IIB theory were Majorana and anti-Majorana respectively. See the Addendum section 7 for a detailed discussion of our convention changes in this current version.
} 
where the superscripts on the $Q_{m n}$ and $P_{m n}$ indicate the degrees of the R-R field strengths involved. The complete expressions for $Q_{m n}$ and $P_{m n}$ are obtained by summing the various NS-NS and R-R contributions listed in (3.13) and (3.14) above.

We now implement the T-duality transformations for the $O\left(\theta^{2}\right)$ backgrounds, as given in (3.9). Specifically, this means that we should take the expressions given by (3.13) and (3.14), apply the transformations (3.9), and then seek to re-interpret the resulting tilded quantities as the $9+1$ decompositions of fully ten-dimensional covariant tilded backgrounds $\widetilde{P}_{m n}$ and $\widetilde{Q}_{m n}$. It is helpful to study the R-R terms, given in (3.14), first. It is straightforward to see that the last step, of re-interpreting the transformed nine-dimensional backgrounds as the reductions of covariant ten-dimensional ones, is impossible if one tries still to use the original two $\theta^{A}$ fermionic coordinates of the type IIA action (2.8). However, if one uses instead the $\tilde{\theta}^{A}$ fermionic coordinates, then the transformed nine-dimensional backgrounds can indeed be expressed as the reductions of covariant ten-dimensional ones. To see how this works, let us consider a sample calculation in detail.

The dimensional reduction of $Q_{m n}^{(4)}$ gives rise to a number of terms, including the contribution to $Q_{m^{\prime} 0}$ of the 3 -form $F_{m^{\prime} n^{\prime} p^{\prime}}$ that comes from $F_{m n p q}$. From (3.14), we see that this contribution will be

$$
Q_{m^{\prime} 0}^{(4)}=\frac{\mathrm{i}}{12} e^{\phi} \bar{\theta}^{(1} \Gamma_{m^{\prime}} p^{\prime} q^{\prime} r^{\prime} \theta^{2)} F_{p^{\prime} q^{\prime} r^{\prime} 0}+\cdots
$$

In terms of the type IIB spinors this can be seen to be

$$
Q_{m^{\prime} 0}^{(4)}=\frac{\mathrm{i}}{12} e^{\frac{3}{4} \phi-\frac{\sqrt{7}}{4} \varphi} \overline{\tilde{\theta}}^{[1} \Gamma_{m^{\prime}}{ }^{\prime^{\prime} q^{\prime} r^{\prime}} \Gamma_{0} \tilde{\theta}^{2]} F_{p^{\prime} q^{\prime} r^{\prime}}+\cdots
$$

where the changed dilaton prefactor results from the conversion of the $D=10$ tangent-space components of $F_{p^{\prime} q^{\prime} r^{\prime} 0}$ to the $D=9$ tangent-space components of $F_{p^{\prime} q^{\prime} r^{\prime}}$. From (3.9), $Q_{m^{\prime} 0}^{(4)}$ is related to the $\left(m^{\prime} 0\right)$ components of an antisymmetric term $\widetilde{P}_{m n}$ in the T-duality transformed theory. One can easily see that it arises from the reduction of the ten-dimensional quantity

$$
\widetilde{P}_{m n}^{(3)}=-\frac{\mathrm{i}}{12} e^{\frac{3}{4} \phi-\frac{\sqrt{7}}{4} \varphi} \overline{\tilde{\theta}}^{[1} \Gamma_{[m} \Gamma^{p q r} \Gamma_{n]} \tilde{\theta}^{2]} \widetilde{F}_{p q r}
$$

where $\widetilde{F}_{p^{\prime} q^{\prime} r^{\prime}}=-F_{p^{\prime} q^{\prime} r^{\prime}}$. (The need for the minus sign becomes apparent after following a complete chain of analogous T-duality transformations.) In fact if we look at the complete set of T-duality transformations, we find that the scalars $\phi$ and $\varphi$ occur in the same

\footnotetext{
${ }^{6} \mathrm{It}$ is worth emphasising that this requirement of reinterpreting the T-duality transformed type IIA string action as a covariant ten-dimensional action allows one to deduce the existence of the type IIB string with its chiral fermions and self-dual 5-form.
} 
combination $\frac{3}{4} \phi-\frac{\sqrt{7}}{4} \varphi$ in all the $\widetilde{P}_{m n}$ and $\widetilde{Q}_{m n}$ expressions. Noting that in the T-duality transformed theory there should be a single scalar $\tilde{\phi}$ in $D=10$, it is therefore natural to define it to be

$$
\tilde{\phi}=\frac{3}{4} \phi-\frac{\sqrt{7}}{4} \varphi .
$$

We now recall that in the Kaluza-Klein reduction (3.7) for the metric of the T-transformed theory, the $\tilde{g}_{00}$ component was parametrized in terms of $\tilde{\lambda}$, which was related to $\phi$ and $\varphi$ by $\tilde{\lambda}=-\frac{1}{4} \phi-\frac{\sqrt{7}}{4} \varphi($ see $(3.8)$ ). Since this is not orthogonal to $\tilde{\phi}$, it is natural to reparametrize it in terms of $\tilde{\phi}$ and a second linear combination $\tilde{\varphi}$ of $\phi$ and $\varphi$ that is orthogonal to $\tilde{\phi}$, namely

$$
\tilde{\varphi}=-\frac{\sqrt{7}}{4} \phi-\frac{3}{4} \varphi
$$

In terms of these two orthogonal fields, we now have $\tilde{\lambda}=\frac{1}{4} \tilde{\phi}+\frac{\sqrt{7}}{4} \tilde{\varphi}$. This is identical in form to the original untilded $\lambda$ in the Kaluza-Klein metric decomposition (3.7) for the type IIA theory. The relations (3.18) and (3.19) which we have derived here are precisely the transformations that relate the dilatonic scalars in the nine-dimensional reductions of the type IIA and type IIB supergravities (see, for example, [12]).

It is interesting to note that our derivation of the relations (3.18) and (3.19) in the present Green-Schwarz formalism was a purely classical one. By contrast, in a standard NSR sigma model formulation of T-duality, the derivation of the dilaton transformations requires a detailed consideration of how conformal invariance can be preserved under quantisation [1. 2]. The essential difference in the Green-Schwarz formalism that has allowed us to obtain the dilaton transformations from purely algebraic classical considerations is the presence of R-R background field strengths in the string action.

After carrying out the entire chain of T-duality transformations (3.9), involving repeated steps paralleling those that we have illustrated above, we find that the R-R couplings in $D=10$ become

$$
\begin{aligned}
\widetilde{Q}_{m n}^{(1)} & =-\frac{\mathrm{i}}{2} e^{\tilde{\phi}} \overline{\tilde{\theta}}^{[1} \Gamma_{(m} \Gamma^{p} \Gamma_{n)} \tilde{\theta}^{2]} \widetilde{F}_{p}, \\
\widetilde{P}_{m n}^{(1)} & =\frac{\mathrm{i}}{2} e^{\tilde{\phi}} \overline{\tilde{\theta}}^{(1} \Gamma_{[m} \Gamma^{p} \Gamma_{n]} \tilde{\theta}^{2)} \widetilde{F}_{p}, \\
\widetilde{Q}_{m n}^{(3)} & =\frac{\mathrm{i}}{12} e^{\tilde{\phi}} \overline{\tilde{\theta}}^{(1} \Gamma_{(m} \Gamma^{p q r} \Gamma_{n)} \tilde{\theta}^{2)} \widetilde{F}_{p q r}, \\
\widetilde{P}_{m n}^{(3)} & =-\frac{\mathrm{i}}{12} e^{\tilde{\phi}} \overline{\tilde{\theta}}^{[1} \Gamma_{[m} \Gamma^{p q r} \Gamma_{n]} \tilde{\theta}^{2]} \widetilde{F}_{p q r}, \\
\widetilde{Q}_{m n}^{(5)} & =-\frac{\mathrm{i}}{480} e^{\tilde{\phi}} \overline{\tilde{\theta}}^{[1} \Gamma_{(m} \Gamma^{p_{1} \cdots p_{5}} \Gamma_{n)} \tilde{\theta}^{2]} \widetilde{F}_{p_{1} \cdots p_{5}}, \\
\widetilde{P}_{m n}^{(5)} & =\frac{\mathrm{i}}{480} e^{\tilde{\phi}} \overline{\tilde{\theta}}^{(1} \Gamma_{[m} \Gamma^{p_{1} \cdots p_{5}} \Gamma_{n]} \tilde{\theta}^{2)} \widetilde{F}_{p_{1} \cdots p_{5}} .
\end{aligned}
$$


Note that the chirality of the $\tilde{\theta}^{A}$ fermions implies that the 5 -form $\widetilde{F}_{(5)}$ is self-dual. Note also that $\widetilde{F}_{(1)}=d \chi$, where $\chi$ is the axionic scalar of the type IIB theory.

We find that the type IIB 1-form, 3-form and 5-form field strengths appearing in (3.20) are related to the 2 -form and 4 -form field strengths of the type IIA action (2.8) as follows. Reducing the $D=10$ type IIA fields as

$$
F_{(4)} \longrightarrow F_{(4)}+F_{(3)} \wedge\left(d Y+\mathcal{A}_{(1)}\right), \quad F_{(2)} \longrightarrow F_{(2)}+F_{(1)} \wedge\left(d Y+\mathcal{A}_{(1)}\right)
$$

and the $D=10$ type IIB fields as

$$
\begin{aligned}
& \widetilde{F}_{(3)} \longrightarrow \widetilde{F}_{(3)}+\widetilde{F}_{(2)} \wedge\left(d Z+\widetilde{\mathcal{A}}_{(1)}\right), \quad \widetilde{F}_{(1)} \longrightarrow \widetilde{F}_{(1)}, \\
& \widetilde{F}_{(5)} \longrightarrow \widetilde{F}_{(5)}+\widetilde{F}_{(4)} \wedge\left(d Z+\widetilde{\mathcal{A}}_{(1)}\right),
\end{aligned}
$$

where $\widetilde{F}_{(5)}$ and $\widetilde{F}_{(4)}$ in $D=9$ are related by Hodge duality, we find that the nine-dimensional R-R fields must be identified as follows:

$$
\widetilde{F}_{(4)}=F_{(4)}, \quad \widetilde{F}_{(3)}=-F_{(3)}, \quad \widetilde{F}_{(2)}=F_{(2)}, \quad \widetilde{F}_{(1)}=-F_{(1)} .
$$

This particular pattern of identifications, including the alternating signs, is precisely in agreement with the results for R-R T-duality that one finds at the field-theory level. (See, for example, [12].)

The discussion of the T-duality relations for the NS-NS contributions at $O\left(\theta^{2}\right)$ proceeds in an analogous fashion. Following a similar strategy to that described above, we find that the various background fields can be rewritten, after applying the T-duality transformations (3.8), in the $D=10$ covariant forms

$$
\begin{aligned}
\widetilde{Q}_{m n}^{\mathrm{NS}} & =\frac{\mathrm{i}}{4}\left[\overline{\tilde{\theta}}^{1} \Gamma_{(m}^{p q} \tilde{\theta}^{1}-\overline{\tilde{\theta}}^{2} \Gamma_{(m}^{p q} \tilde{\theta}^{2}\right] \widetilde{F}_{n) p q}^{\mathrm{NS}}+\frac{\mathrm{i}}{2}\left[\overline{\tilde{\theta}}^{1} \Gamma_{(m} \Gamma_{|p q|} \tilde{\theta}^{1}+\overline{\tilde{\theta}}^{2} \Gamma_{(m} \Gamma_{|p q|} \tilde{\theta}^{2}\right] \tilde{\omega}_{n)}{ }^{p q}, \\
\widetilde{P}_{m n}^{\mathrm{NS}} & =-\frac{\mathrm{i}}{4}\left[\overline{\tilde{\theta}}^{1} \Gamma_{(m}{ }^{p q} \tilde{\theta}^{1}+\overline{\tilde{\theta}}^{2} \Gamma_{(m}{ }^{p q} \tilde{\theta}^{2}\right] \widetilde{F}_{n) p q}^{\mathrm{NS}}-\frac{\mathrm{i}}{2}\left[\overline{\tilde{\theta}}^{1} \Gamma_{(m} \Gamma_{|p q|} \tilde{\theta}^{1}-\overline{\tilde{\theta}}^{2} \Gamma_{(m} \Gamma_{|p q|} \tilde{\theta}^{2}\right] \tilde{\omega}_{n)}{ }^{p q}, \\
\widetilde{\mathcal{G}}_{i m} & =-\mathrm{i}\left[\overline{\tilde{\theta}}^{1} \Gamma_{m} \partial_{i} \tilde{\theta}^{1}+\overline{\tilde{\theta}}^{2} \Gamma_{m} \partial_{i} \tilde{\theta}^{2}\right], \\
\widetilde{\mathcal{B}}_{i m} & =-\mathrm{i}\left[\overline{\tilde{\theta}}^{1} \Gamma_{m} \partial_{i} \tilde{\theta}^{1}-\overline{\tilde{\theta}}^{2} \Gamma_{m} \partial_{i} \tilde{\theta}^{2}\right],
\end{aligned}
$$

Reducing the ten-dimensional NS-NS fields to $D=9$ in the standard Kaluza-Klein style, with $F_{(3)}^{\mathrm{NS}} \longrightarrow F_{(3)}^{\mathrm{NS}}+F_{(2)}^{\mathrm{NS}} \wedge d z$, we find that here, as in the previous $O\left(\theta^{0}\right)$ discussion, the nine-dimensional type IIA and type IIB NS-NS fields are related in the standard way, with

$$
\widetilde{F}_{(2)}^{\mathrm{NS}}=\mathcal{F}_{(2)}, \quad \widetilde{\mathcal{F}}_{(2)}=F_{(2)}^{\mathrm{NS}},
$$

where $\mathcal{F}_{(2)}=d \mathcal{A}_{(1)}$ and $\widetilde{\mathcal{F}}_{(2)}=d \mathcal{A}_{(1)}$. This is just the standard interchange of Kaluza-Klein and winding vectors. 
A number of comments are now in order. Firstly, we may observe by comparing (3.13) and (3.24) that the NS-NS terms at $O\left(\theta^{2}\right)$ are identical in form in the type IIA and type IIB Green-Schwarz actions. This is in accordance with what one would expect; it was already seen, of course, at $O\left(\theta^{0}\right)$. A particular consequence of this is that the $\partial_{i} \tilde{\theta}^{A}$ terms and the $\frac{1}{4} \partial_{i} X^{\mu} \tilde{\omega}_{\mu}^{m n} \Gamma_{m n} \tilde{\theta}^{A}$ terms, which were separated into contributions in $Q_{m n}^{\mathrm{NS}}, P_{m n}^{\mathrm{NS}}$, $\mathcal{G}_{i m}$ and $\mathcal{B}_{i m}$ during the implementation of the T-duality transformation, have re-assembled themselves to make the spacetime Lorentz-covariant derivative $D_{i} \tilde{\theta}^{A}$ again.

A second observation is that in the R-R sector, the expressions for $Q_{m n}$ and $P_{m n}$ in the type IIA theory in (3.14) and in the type IIB theory (3.20) all have the same structural form. In all cases, the general structure for the coupling to a $p$-form is

$$
Q_{m n}^{(p)} \sim \frac{\mathrm{i}}{2 p !} e^{\phi} \bar{\theta}^{1} \Gamma_{(m} \Gamma^{q_{1} \cdots q_{p}} \Gamma_{n)} \theta^{2} F_{q_{1} \cdots q_{p}}, \quad P_{m n}^{(p)} \sim \frac{\mathrm{i}}{2 p !} e^{\phi} \bar{\theta}^{1} \Gamma_{[m} \Gamma^{q_{1} \cdots q_{p}} \Gamma_{n]} \theta^{2} F_{q_{1} \cdots q_{p}} .
$$

(In the case of the 5-form field strength in the type IIB theory, the proper handling of the self-duality constraint implies, as usual, that this term should enter with $\frac{1}{2}$ of the canonical coefficient for a $p$-form.)

To close this section, we may summarise our results for the ten-dimensional type IIB Green-Schwarz action, which takes the form]

$$
\begin{aligned}
\widetilde{\mathcal{L}}_{2}= & -\frac{1}{2} \sqrt{-h} h^{i j} \partial_{i} X^{\mu} \partial_{j} X^{\nu} \widetilde{g}_{\mu \nu}+\frac{1}{2} \epsilon^{i j} \partial_{i} X^{\mu} \partial_{j} X^{\nu} \widetilde{A}_{\mu \nu} \\
& -\mathrm{i} \sqrt{-h} h^{i j}\left(\overline{\tilde{\theta}}^{1} \Gamma_{\mu} D_{j} \tilde{\theta}^{1}+\overline{\tilde{\theta}}^{2} \Gamma_{\mu} D_{j} \tilde{\theta}^{2}\right) \partial_{i} X^{\mu}-\mathrm{i} \epsilon^{i j}\left(\overline{\tilde{\theta}}^{1} \Gamma_{\mu} D_{j} \tilde{\theta}^{1}-\overline{\tilde{\theta}}^{2} \Gamma_{\mu} D_{j} \tilde{\theta}^{2}\right) \partial_{i} X^{\mu} \\
& -\frac{i}{8} \sqrt{-h} h^{i j} \partial_{i} X^{\mu} \partial_{j} X^{\nu}\left(\overline{\tilde{\theta}}^{1} \Gamma_{\mu}^{\rho \sigma} \tilde{\theta}^{1}-\overline{\tilde{\theta}}^{2} \Gamma_{\mu}^{\rho \sigma} \tilde{\theta}^{2}\right) \widetilde{F}_{\nu \rho \sigma}^{\mathrm{NS}} \\
& -\frac{\mathrm{i}}{\epsilon} \epsilon^{i j} \partial_{i} X^{\mu} \partial_{j} X^{\nu}\left(\overline{\tilde{\theta}}^{1} \Gamma_{\mu}^{\rho \sigma} \tilde{\theta}^{1}+\overline{\tilde{\theta}}^{2} \Gamma_{\mu}^{\rho \sigma} \tilde{\theta}^{2}\right) \widetilde{F}_{\nu \rho \sigma}^{\mathrm{NS}}+\mathrm{i} e^{\tilde{\phi}} \partial_{i} X^{\mu} \partial_{j} X^{\nu} \times \\
& {\left[\sqrt{-h} h^{i j}\left(\frac{1}{4} \overline{\tilde{\theta}}^{[1} \Gamma_{\mu} \Gamma^{\rho} \Gamma_{\nu} \tilde{\theta}^{2]} \partial_{\rho} \chi-\frac{1}{24} \overline{\tilde{\theta}}^{(1} \Gamma_{\mu} \Gamma^{\rho \sigma \lambda} \Gamma_{\nu} \tilde{\theta}^{2)} \widetilde{F}_{\rho \sigma \lambda}+\frac{1}{960} \overline{\tilde{\theta}}^{[1} \Gamma_{\mu} \Gamma^{\rho_{1} \cdots \rho_{5}} \Gamma_{\nu} \tilde{\theta}^{2]} \widetilde{F}_{\rho_{1} \cdots \rho_{5}}\right)\right.} \\
& \left.+\epsilon^{i j}\left(\frac{1}{4} \overline{\tilde{\theta}}^{(1} \Gamma_{\mu} \Gamma^{\rho} \Gamma_{\nu} \tilde{\theta}^{2)} \partial_{\rho} \chi-\frac{1}{24} \overline{\tilde{\theta}}^{[1} \Gamma_{\mu} \Gamma^{\rho \sigma \lambda} \Gamma_{\nu} \tilde{\theta}^{2]} \widetilde{F}_{\rho \sigma \lambda}+\frac{1}{960} \overline{\tilde{\theta}}^{(1} \Gamma_{\mu} \Gamma^{\rho_{1} \cdots \rho_{5}} \Gamma_{\nu} \tilde{\theta}^{2)} \widetilde{F}_{\rho_{1} \cdots \rho_{5}}\right)\right] .
\end{aligned}
$$

\section{Green-Schwarz Action for the Massive Type IIA Theory}

It has been shown at the level of the low-energy effective supergravities that the massive type IIA theory and the standard type IIB theory are related via a T-duality transformation that differs from the usual one only in that a generalised Scherk-Schwarz reduction ansatz is now introduced for the axion $\chi$ of the IIB theory [10]. In other words, one makes the

\footnotetext{
${ }^{7}$ We have corrected one typographical sign error that arose in the version of this paper in Nucl. Phys. B573 (2000) 149 and v1. of hep-th/9907202, in the $\epsilon^{i j}$ term on the second line. We have also implemented the modified definition where $\tilde{\theta}^{2}$ is Majorana, as discussed in the Addendum section 7 .
} 
more general Kaluza-Klein ansatz

$$
\chi(x, z) \longrightarrow \chi(x)+m z
$$

when reducing from $D=10$ to $D=9$, while keeping all other ansätze unchanged. Despite the linear dependence on $z$ in (4.1), the usual requirement of $z$-independence of the higherdimensional action and field strengths is still satisfied, in view of the global shift symmetry under $\chi \longrightarrow \chi+$ constant.

It is straightforward to implement the analogue of this generalised reduction in the Tduality transformation of the previous section. Thus we now replace the reduction procedure for $\widetilde{F}_{(1)}$ given in $(3.22)$ by

$$
\widetilde{F}_{(1)} \longrightarrow \widetilde{F}_{(1)}+m\left(d Z+\widetilde{\mathcal{A}}_{(1)}\right) .
$$

(As a consequence, $\widetilde{F}_{(1)}$ in $D=9$ is now given by $\widetilde{F}_{(1)}=d \chi-m \widetilde{\mathcal{A}}_{(1)}$.) We can now use the T-duality rules (3.9) in the direction opposite to that which we followed previously, to deduce the form of the new terms $Q_{m n}^{(0)}$ and $P_{m n}^{(0)}$ that we shall acquire in the type IIA string action. As usual, we find that after applying the T-duality transformation, the ninedimensional expressions can indeed be lifted to covariant ten-dimensional ones. By this means, we obtain the following new terms in the ten-dimensional type IIA Green-Schwarz action:

$$
Q_{m n}^{(0)}=\frac{\mathrm{i}}{2} m e^{\phi}\left(\bar{\theta}^{1} \Gamma_{(m} \Gamma_{n)} \theta^{1}+\bar{\theta}^{2} \Gamma_{(m} \Gamma_{n)} \theta^{2}\right), \quad P_{m n}^{(0)}=\frac{\mathrm{i}}{2} m e^{\phi}\left(\bar{\theta}^{1} \Gamma_{m n} \theta^{1}-\bar{\theta}^{2} \Gamma_{m n} \theta^{2}\right)
$$

(In the expression for $Q_{m n}^{(0)}$ we could, of course, replace $\Gamma_{(m} \Gamma_{n)}$ by $\eta_{m n}$.) Thus up to $\left(O\left(\theta^{2}\right)\right.$, we find that the Green-Schwarz action for the massive type IIA string is given by

$$
\mathcal{L}_{2}^{\text {massive }}=\mathcal{L}_{2}-\frac{\mathrm{i}}{4} m \partial_{i} X^{\mu} \partial_{j} X^{\nu} e^{\phi} \bar{\theta}\left(\sqrt{-h} h^{i j}-\epsilon^{i j} \Gamma_{11}\right) \Gamma_{\mu} \Gamma_{\nu} \theta,
$$

where $\mathcal{L}_{2}$ is given in $(2.8)$, but with the field strengths now given by

$$
\begin{aligned}
& F_{(2)}=d A_{(1)}+m A_{(2)}, \quad F_{(3)}=d A_{(2)}, \\
& F_{(4)}=d A_{(3)}+A_{(1)} \wedge d A_{(2)}+\frac{1}{2} m A_{(2)} \wedge A_{(2)} .
\end{aligned}
$$

It is often more appropriate to use a formalism where the mass term of the IIA theory, which can be thought of as a 0-form field strength, is replaced by its dual 10-form field strength. In this case the action will be given instead by

$$
\mathcal{L}_{2}^{\text {massive }}=\mathcal{L}_{2}-\frac{\mathrm{i}}{4 \times 10 !} \partial_{i} X^{\mu} \partial_{j} X^{\nu} e^{\phi} \bar{\theta}\left(\sqrt{-h} h^{i j}-\epsilon^{i j} \Gamma_{11}\right) \Gamma_{\mu} \Gamma^{\rho_{1} \cdots \rho_{10}} \Gamma_{\nu} \theta F_{\rho_{1} \cdots \rho_{10}} .
$$




\section{Massless/massive type IIA T-duality}

In the previous section, we derived the T-duality transformation between the type IIA and type IIB Green-Schwarz actions. In particular, if the 0 -form $m$ vanishes, this relates the massless type IIA and type IIB theories. When $m$ is non-vanishing, the T-duality maps from type IIB to the massive type IIA string theory. This T-duality has been discussed in Refs [10, 13, 11, 12] at the level of the low-energy effective action, by considering the Scherk-Schwarz reduction of type IIB supergravity. This leads to the expectation that there should be a T-duality that directly relates the massless and massive type IIA string theories. This issue was explored in [14, by considering the T-duality between the D8-brane and the D6-brane in eight dimensions. Since the D6-brane in $D=8$ has, from an eleven-dimensional point of view, the internal structure of either a circle bundle over a 2-torus [20] or a 2-torus bundle over a circle [14], this would provide a geometrical relation between M-theory and the massive type IIA string.

Here, we shall consider the T-duality relations between the massive and massless type IIA strings in detail, making use of the results of the previous sections. In particular, we have seen that the results of T-duality transformations on the string variables $\left(X^{\mu}, \theta\right)$ are implemented on the background fields by means of standard nonlinear supergravity global symmetry transformations. This would then also imply that a T-duality map between an original massless string theory (with an appropriate non-trivial R-R background) and the corresponding image massive theory will similarly be implemented by a backgroundfield transformation that can be read off from previous work on effective field theories. In particular, for the purposes of the present discussion we shall consider string theories on backgrounds with two isometries, which are related to previous work on $D=8$ effective supergravities.

Accordingly, let us consider massless type IIA string theory on a background with two $U(1)$ isometries, namely where all spacetime fields are independent of two spacetime coordinates $Y^{a}, a=1,2$. Defining $V_{i}^{a}=\partial_{i} Y^{a}$, we introduce the Lagrange multiplier term

$$
\mathcal{L}=\mathcal{L}_{0}+\epsilon^{i j} \partial_{i} Z_{a} V_{j}^{a}
$$

Next, we integrate out the two $V_{i}^{a}$. Two possible cases arise, according to whether or not the 2 -form field strength $F_{(2)}$ with indices projected into the compactified directions vanishes. These two cases correspond to massless or massive string backgrounds respectively.

Consider first the massless case. After performing a T-duality transformation by integrating out the two $V_{i}^{a}$, the Lagrangian can once again be put into the form of the type IIA 
massless string action, but with background fields now subjected to a transformation which can be identified as one of the perturbative symmetries of the $D=8$ effective supergravity theory. After a final relabelling of the Lagrange multipliers $\left(Z_{1}=\tilde{Y}^{2}, Z_{2}=-\tilde{Y}^{1}\right)$, this "double" T-duality transformation corresponds to an inversion and interchange of the two toroidal radii; $R_{1} \longrightarrow 1 / R_{2}$ and $R_{2} \longrightarrow 1 / R_{1}$. Unlike the situation where one performs a T-duality inversion on just a single circle, which maps the type IIA theory into the IIB theory, this double T-duality inversion maps the type IIA theory back into itself. In other words, the transformed theory can still be lifted up to the type IIA massless string action in $D=10$, so this constitutes a genuine element of the symmetry group of the type IIA string.

As with the single-inversion T-duality discussed in section 3 , the effect of the doubleinversion T-duality on the background fields is identical to a standard transformation of the corresponding lower-dimensional effective field theory. In the single-inversion case, this was the well-known redefinition (not a symmetry) that relates the fields of the type IIA and type IIB forms of nine-dimensional supergravity. In the double-inversion case, the corresponding field-theory transformation is a symmetry of the eight-dimensional supergravity, which is contained in the perturbative T-duality $S O(2,2)$ subgroup of the $S L(3, \mathbb{R}) \times S L(2, \mathbb{R})$ Cremmer-Julia symmetry of maximal eight-dimensional supergravity.

Before we identify which $D=8$ supergravity symmetry transformation the T-duality transformation produces, let us recall the structure of the T-duality symmetry group. The perturbative T-duality group that arises for spacetimes with the isometries of a $d$-torus is $S O(d, d)$. This is a subgroup of the Cremmer-Julia supergravity symmetry group for the effective field theory obtained by dimensionally reducing from $D=10$ on such a torus; in the case of $D=8$ maximal supergravity, the Cremmer-Julia symmetry group is $S L(3, \mathbb{R}) \times$ $S L(2, \mathbb{R})$. The corresponding $S O(2,2)$ perturbative symmetry is isomorphic to $S L(2, \mathbb{R})_{1} \times$ $S L(2, \mathbb{R})_{2}$, where the $S L(2, \mathbb{R})_{2}$ factor is the second factor in the Cremmer-Julia group and the $S L(2, \mathbb{R})_{1}$ factor is a subgroup of the Cremmer-Julia $S L(3, \mathbb{R}) . \quad S L(2, \mathbb{R})_{1}$ has its origin in general coordinate transformations of the 2-torus. In fact, the full residuum of

\footnotetext{
${ }^{8}$ Note that in the case of bosonic or type I strings, this single-inversion transformation is a symmetry, and it enlarges the total T-duality group from $S O(2,2)$ to $O(2,2)$. See 21] for a recent discussion, at the level of supergravity action, of how R-R potentials transform under the $S O(d, d)$ group.

${ }^{9}$ Since we shall be discussing symmetry groups both in their continuous forms at the level of the effective field theories, and in their discretised forms in the string theories, we shall tend to refer to them always in their continuous forms, with their discretisations to integer coefficients in the string-theory context being understood.
} 
the "internal" general coordinate transformations is $G L(2, \mathbb{R}) \sim \mathbb{R} \times S L(2, \mathbb{R})$, but the $\mathbb{R}$ factor becomes part of $S L(2, \mathbb{R})_{2}$. The remaining two $S L(2, \mathbb{R})_{2}$ generators arise from an $O(2)$ transformation independent of the general-coordinate $G L(2, \mathbb{R})$ and from constant shifts of the axion $\chi=A_{(0)}$ that comes from the internal components of the NS-NS 2-form in $D=10$. (See, for example, [22] for a discussion of $O(d, d)$ T-duality for string actions on nontrivial NSR backgrounds.)

Now we shall identify which $S O(2,2)$ transformation has been generated by the doubleinversion T-duality. Specifically, we shall show that it is the element of the $S L(2, \mathbb{R})_{2}$ factor represented by the matrix

$$
\Lambda=\left(\begin{array}{cc}
0 & 1 \\
-1 & 0
\end{array}\right)
$$

To see how this works, let us consider the double-inversion T-duality transformation in detail. We already saw in section 3 that the usual presentation of the single-inversion T-duality transformation leads to rather opaque transformation rules of the form (3.5), which assume the more elegant form (3.8) when the ten-dimensional fields are decomposed in terms of nine-dimensional fields in a geometrically-natural way. Specifically, it is the standard Kaluza-Klein ansatz that provides this natural framework for this decomposition. This advantage becomes all the more persuasive in the present context, where we wish to implement the double-inversion T-duality transformation corresponding to integrating out both of the auxiliary fields $V_{i}^{a}$ in (5.1). Thus we begin by writing the ten-dimensional string metric in the Kaluza-Klein decomposition appropriate to the assumed form where there are two $U(1)$ isometries on a 2-torus:

$$
d \hat{s}_{10}^{2}=d s_{8}^{2}+e^{-f+\psi}\left(d Y^{1}+\mathcal{A}_{(1)}^{1}+\mathcal{A}_{(0) 2}^{1} d Y^{2}\right)^{2}+e^{-f-\psi}\left(d Y^{2}+\mathcal{A}_{(1)}^{2}\right)^{2},
$$

where all fields are independent of the two toroidal coordinates $\left(Y^{1}, Y^{2}\right)$, and we have defined

$$
f \equiv-\frac{1}{2} \phi-\frac{3}{2 \sqrt{7}} \varphi_{1}-\sqrt{\frac{3}{7}} \varphi_{2}, \quad \psi \equiv \frac{2}{\sqrt{7}} \varphi_{1}-\sqrt{\frac{3}{7}} \varphi_{2} .
$$

Here, $\varphi_{1}$ and $\varphi_{2}$ are the usual dilatonic scalars coming from the reduction steps to $D=9$ and $D=8$ respectively. As we shall see, the combination $f$ will be the canonicallynormalised dilaton in the $S L(2, \mathbb{R}) / O(2)$ scalar coset describing the $S L(2, \mathbb{R})_{2}$ global symmetry. The combination $\psi$, also canonically normalised, is orthogonal to $f$ and lives in the $S L(3, \mathbb{R}) / O(3)$ part of the total scalar manifold. We also make a standard Kaluza-Klein decomposition for the 2 -form potential $A_{\mu \nu}$ :

$$
\hat{A}_{(2)}=A_{(2)}+A_{(1) a} \wedge d Y^{a}+\chi d Y^{1} \wedge d Y^{2}
$$


In fact $\chi$ will turn out to be the axion in the $S L(2, \mathbb{R})_{2} / O(2)$ scalar coset.

We are now ready to implement the double-inversion T-duality transformation. Thus we begin with the sigma-model Lagrangian

$$
\mathcal{L}_{0}=-\frac{1}{2} \sqrt{-h} h^{i j} \partial_{i} X^{\mu} \partial_{j} X^{\nu} G_{\mu \nu}+\frac{1}{2} \epsilon^{i j} \partial_{i} X^{\mu} \partial_{j} X^{\nu} A_{\mu \nu}
$$

and make the assumption that all fields are independent of $Y^{a}=\left(Y^{1}, Y^{2}\right)$, where we decompose the coordinates as $X^{\mu}=\left(X^{\mu^{\prime}}, Y^{a}\right)$. Then replacing $\partial_{i} Y^{a}$ by $V_{i}^{a}$, adding the Lagrange multiplier term as in (5.1), and integrating out the two $V_{i}^{a}$, we arrive at a T-duality transformed Lagrangian which again has the same general form as (5.6), with $\left(Z_{1}, Z_{2}\right)$ now playing the rôles of the two toroidal coordinates. In fact, it is natural to relabel these in terms of two tilded coordinates $\tilde{Y}^{a}$, according to the rule $Z_{1}=\tilde{Y}^{2}, Z_{2}=-\tilde{Y}^{1}$. As we indicated above, at the intermediate stages of the calculation the results are cumbersome. However, they become rather simple when expressed in terms of the fields appearing in the Kaluza-Klein decomposition, and we shall present only these final results here. To do so, it is useful first to make the following redefinitions:

$$
\begin{aligned}
& \mathcal{A}_{(1)}^{\prime 1}=\mathcal{A}_{(1)}^{1}-\mathcal{A}_{(0) 2}^{1} \mathcal{A}_{(1)}^{2}, \quad A_{(1) 1}^{\prime}=A_{(1) 1}+\chi \mathcal{A}_{(1)}^{2}, \\
& A_{(1) 2}^{\prime}=A_{(1) 2}-\chi \mathcal{A}_{(1)}^{1}{ }^{\prime}, \quad A_{(2)}^{\prime}=A_{(2)}-\chi \mathcal{A}_{(1)}^{1}{ }^{\prime} \wedge \mathcal{A}_{(1)}^{2} .
\end{aligned}
$$

After the double-inverse T-duality transformation, we find that the sigma-model Lagrangian can be recast in the form (5.6), using tilded fields which are related to the original untilded ones as follows. First of all, in the scalar sector we find

$$
\begin{aligned}
e^{-\tilde{f}} & =\frac{e^{-f}}{e^{-2 f}+\chi^{2}}, \quad \tilde{\chi}=-\frac{\chi}{e^{-2 f}+\chi^{2}}, \\
\widetilde{\psi} & =\psi, \quad \widetilde{\mathcal{A}}_{(0) 2}^{1}=\mathcal{A}_{(0) 2}^{1} .
\end{aligned}
$$

This shows that the dilaton $f$ and the axion $\chi$ have transformed under precisely the $S L(2, \mathbb{R})$ matrix $\Lambda$ given in (5.2), where $\Lambda=\left(\begin{array}{ll}a & b \\ c & d\end{array}\right)$ acts by fractional linear transformations $\tilde{\tau}=(a \tau+b) /(c \tau+d)$ on $\tau \equiv \chi+\mathrm{i} e^{-f}$. On the other hand the other dilatonic scalar combination $\psi$, and the axion $\mathcal{A}_{(0) 2}^{1}$ associated with $S L(2, \mathbb{R})_{1}$, are inert. We find that the remaining fields are transformed in the following way:

$$
\begin{aligned}
\widetilde{\mathcal{A}}_{(1)}^{\prime 1} & =-A_{(1) 2}^{\prime}, \quad \widetilde{\mathcal{A}}_{(1)}^{2}=A_{(1) 1}^{\prime}, \\
\widetilde{A}_{(1) 1}^{\prime} & =-\mathcal{A}_{(1)}^{2}, \quad \widetilde{A}_{(1) 2}^{\prime}=\mathcal{A}_{(1)}^{1}{ }^{\prime}, \\
\widetilde{A}_{(2)}^{\prime} & =A_{(2)}^{\prime}+\mathcal{A}_{(1)}^{1}{ }^{\prime} \wedge A_{(1) 1}^{\prime}+\mathcal{A}_{(1)}^{2} \wedge A_{(1) 2}^{\prime}, \\
\tilde{g}_{\mu^{\prime} \nu^{\prime}} & =g_{\mu^{\prime} \nu^{\prime}} .
\end{aligned}
$$


Note that here $g_{\mu^{\prime} \nu^{\prime}}$ denotes the eight-dimensional string-frame metric $d s_{8}^{2}$ appearing in the Kaluza-Klein decomposition (5.3). It is therefore related to $G_{\mu^{\prime} \nu^{\prime}}$ by $g_{\mu^{\prime} \nu^{\prime}}=G_{\mu^{\prime} \nu^{\prime}}+$ $e^{-f}\left(e^{\psi} \mathcal{A}_{\mu^{\prime}}^{1} \mathcal{A}_{\nu^{\prime}}^{1}+e^{-\psi} \mathcal{A}_{\mu^{\prime}}^{2} \mathcal{A}_{\nu^{\prime}}^{2}\right)$.

The transformations (5.8) and (5.9) describe a symmetry of the truncation of the eightdimensional supergravity to its NS-NS sector. In fact, when appropriately augmented by transformations for the R-R fields, it is a symmetry of the full theory (see the appendix for a complete discussion); as we discussed above, it is the $Z_{2}$ subgroup of the $S L(2, \mathbb{R})_{2}$ factor of the T-duality symmetry given in (5.2).

So far, we have demonstrated the massless/massive type IIA T-duality at the level of the NS-NS background fields, in what is a rather natural generalisation of the standard discussion for IIA/IIB duality. Since we are working in the Green-Schwarz formalism, this string-theoretic derivation of massless/massive IIA T-duality can be extended to the R-R sector too, as we did for IIA/IIB T-duality in section 3. To do this, we again start from a generic Lagrangian of the form (3.1), with $G_{\mu \nu}$ and $B_{\mu \nu}$ containing $O\left(\theta^{2}\right)$ terms $Q_{\mu \nu}$ and $P_{\mu \nu}$ as in (3.2), together also with the terms $\mathcal{G}_{j \mu}$ and $\mathcal{B}_{j \mu}$ containing the $\bar{\theta} \partial_{j} \theta$ at $O\left(\theta^{2}\right)$. Following the lessons learned in section 3.1, where we saw that the relations between the lower-dimensional components of the original and the T-duality transformed $O\left(\theta^{2}\right)$ tensors are much simpler if written in terms of tangent-space indices, we follow the same strategy here. After algebra of considerable complexity, we arrive at the following expressions for the tilded $O\left(\theta^{2}\right)$ backgrounds in terms of the original ones:

$$
\begin{aligned}
& \widetilde{Q}_{m^{\prime} n^{\prime}}=Q_{m^{\prime} n^{\prime}}, \quad \widetilde{P}_{m^{\prime} n^{\prime}}=P_{m^{\prime} n^{\prime}}, \quad \widetilde{\mathcal{G}}_{i m^{\prime}}=\mathcal{G}_{i m^{\prime}}, \quad \widetilde{\mathcal{B}}_{i m^{\prime}}=\mathcal{B}_{i m^{\prime}}, \\
& \widetilde{Q}_{m^{\prime} a}=e^{-\frac{1}{2}(\tilde{f}+f)}\left(-\epsilon_{a b} P_{m^{\prime} b}+\chi e^{f} Q_{m^{\prime} a}\right), \\
& \widetilde{P}_{m^{\prime} a}=e^{-\frac{1}{2}(\tilde{f}+f)}\left(-\epsilon_{a b} Q_{m^{\prime} b}+\chi e^{f} P_{m^{\prime} a}\right), \\
& \widetilde{Q}_{12}=Q_{12}, \quad \widetilde{Q}_{11}-\widetilde{Q}_{22}=Q_{11}-Q_{22}, \\
& \widetilde{Q}+\widetilde{\chi} e^{\tilde{f}} \widetilde{P}_{12}=-Q-\chi e^{f} P_{12}, \quad \widetilde{P}_{12}-\tilde{\chi} e^{\tilde{f}} \widetilde{Q}=-P_{12}+\chi e^{f} Q, \\
& \widetilde{\mathcal{G}}_{i a}=e^{-\frac{1}{2}(\tilde{f}+f)}\left(\epsilon_{a b} \mathcal{B}_{i b}+\chi e^{f} \mathcal{G}_{i a}\right), \quad \widetilde{\mathcal{B}}_{i a}=e^{-\frac{1}{2}(\tilde{f}+f)}\left(\epsilon_{a b} \mathcal{G}_{i b}+\chi e^{f} \mathcal{B}_{i a}\right),
\end{aligned}
$$

Our notation here is that $m^{\prime}$ denotes a tangent-space index restricted to the eight-dimensional subspace, and $a$ and $b$ denote tangent-space indices ranging over the two values corresponding to the directions of the two $U(1)$ isometries. The explicit numerical indices 1 and 2 similarly denote these two tangent-space index values. The quantity $Q$ is defined by $Q \equiv \frac{1}{2}\left(Q_{11}+Q_{22}\right)$, and likewise $\widetilde{Q} \equiv \frac{1}{2}\left(\widetilde{Q}_{11}+\widetilde{Q}_{22}\right)$.

In the appendix, we derive the complete $S L(2, \mathbb{R})$ transformations of the NS-NS and $\mathrm{R}-\mathrm{R}$ fields of the eight-dimensional supergravity. In the NS-NS sector, the $Z_{2}$ subgroup 
corresponding to (5.2) coincides precisely with the T-duality relations that we derived in (5.8) and (5.9) by string-theoretic methods. We have verified that this is also true in the $\mathrm{R}-\mathrm{R}$ sector, namely that $Z_{2}$ transformations of the R-R fields agree with the transformations that follow from the $O\left(\theta^{2}\right)$ relations (5.10). To demonstrate this, one has to follow steps analogous to those that we presented for the case of type IIA/IIB T-duality in section 3.2. Note that the fermionic coordinates $\theta$ also undergo a transformation under the double T-duality, with $\tilde{\theta}=e^{-\frac{1}{2} \alpha \Gamma_{11} \Gamma_{1} \Gamma_{2}} \theta$, where $\sin \alpha=e^{-\frac{1}{2} \tilde{f}-\frac{1}{2} f}$. This corresponds to a compensating transformation in the $O(2)$ denominator subgroup of the $S L(2, \mathbb{R})_{2} / O(2)$ coset.

Now let us consider the case where $F_{(0) 12}=m$ is allowed to be a non-vanishing part of the background for the massless type IIA theory. (This corresponds, at the level of the effective field theory, to a Scherk-Schwarz reduction of the axion $A_{(0) 1}$ in $D=9$ that comes from the R-R 1-form $A_{(1)}$ in $D=10$.) After integrating over the two $V_{i}^{a}$ auxiliary fields in (5.1), the resulting Lagrangian no longer allows a direct interpretation as a dimensional reduction of the massless ten-dimensional type IIA string. By this we mean that, unlike in the $F_{(0) 12}=0$ discussion above, the tilded backgrounds of the T-duality transformed theory cannot be directly interpreted as the fields appearing in the given Kaluza-Klein dimensional-reduction ansatz. For example, the tilded 1-forms $\widetilde{\mathcal{A}}_{(1)}^{a}$ and $\widetilde{A}_{(1) a}$, and the tilded dilatonic scalars $\overrightarrow{\tilde{\phi}}$, arising after integrating over the two $V_{i}^{a}$, which in the $F_{(0) 12}=0$ case could respectively be interpreted as the Kaluza-Klein vectors, winding vectors and dilatons in the Kaluza-Klein reduction ansatz for the type IIA theory, cannot be so interpreted once $F_{(0) 12}=m \neq 0$.

The reason why this has happened is that the $S L(2, \mathbb{R})_{2}$ symmetry is broken by the cosmological potential that arises in the Scherk-Schwarz reduction with $F_{(0) 12}=m \neq 0$ [13]. The full Lagrangian is given in the appendix; here, we shall just consider the relevant terms in order to illustrate the point. The cosmological term, together with the kinetic terms for the scalars of the $S L(2, \mathbb{R})_{2} / O(2)$ coset, is given by

$$
\mathcal{L}=-\frac{1}{2} e m^{2} e^{f+\frac{4}{\sqrt{3}} \sigma}-\frac{1}{2} e(\partial f)^{2}-\frac{1}{2} e e^{2 f}(\partial \chi)^{2},
$$

where

$$
\sigma \equiv \frac{\sqrt{3}}{2} \phi-\frac{\sqrt{3}}{2 \sqrt{7}} \varphi_{1}-\frac{1}{\sqrt{7}} \varphi_{2}
$$

is the third linear combination of the three dilatonic scalars; it is canonically normalised and is orthogonal to $f$ and to $\psi$ defined in (5.4). We can think of the cosmological term as being the "kinetic term" for the 0 -form field strength $F_{(0) 12}=m$. The key point that distinguishes this from the case when $m=0$ is that whereas all the other field strengths are 
either invariant under $S L(2, \mathbb{R})_{2}$ or else they occur in doublet pairs, here we have a single term which is not invariant under $S L(2, \mathbb{R})_{2}$, thus breaking the symmetry. Nonetheless, we can still view $S L(2, \mathbb{R})_{2}$ as defining a set of field redefinitions, albeit ones that now change the form of the Lagrangian. In particular, it is easy to see that for a general $S L(2, \mathbb{R})_{2}$ transformation of the backgrounds, for which

$$
e^{-f} \longrightarrow \frac{e^{-f}}{(c \chi+d)^{2}+c^{2} e^{-2 f}}, \quad \chi \longrightarrow \frac{(a \chi+b)(c \chi+d)+a c e^{-2 f}}{(c \chi+d)^{2}+c^{2} e^{-2 f}}
$$

the Lagrangian (5.11) transforms into

$$
\mathcal{L}=-\frac{1}{2} e m_{1}^{2} e^{-f+\frac{4}{\sqrt{3}} \sigma}-\frac{1}{2} e\left(m_{2}+m_{1} \chi\right)^{2} e^{f+\frac{4}{\sqrt{3}} \sigma}-\frac{1}{2} e(\partial f)^{2}-\frac{1}{2} e e^{2 f}(\partial \chi)^{2},
$$

where

$$
\left(\begin{array}{l}
m_{1} \\
m_{2}
\end{array}\right)=\Lambda^{T}\left(\begin{array}{l}
0 \\
m
\end{array}\right),
$$

with $\Lambda=\left(\begin{array}{ll}a & b \\ c & d\end{array}\right)$.

Our specific $S L(2, \mathbb{R})_{2}$ transformation that results from the double-inversion T-duality has $\Lambda$ given by (5.2), and hence the Lagrangian (5.14) becomes

$$
\mathcal{L}=-\frac{1}{2} e m^{2} e^{-f+\frac{4}{\sqrt{3}} \sigma}-\frac{1}{2} e m^{2} \chi^{2} e^{f+\frac{4}{\sqrt{3}} \sigma}-\frac{1}{2} e(\partial f)^{2}-\frac{1}{2} e e^{2 f}(\partial \chi)^{2}
$$

It is clear that this is different from the original form of the Lagrangian (5.11), showing that the mass term breaks not only the general $S L(2, \mathbb{R})_{2}$ symmetry but also the specific $Z_{2}$ subgroup corresponding to the double-inversion T-duality (5.2). However, the T-duality transformed backgrounds do still allow themselves to be directly lifted to a covariant theory in $D=10$, but now it is to the massive IIA theory, rather than to the massless theory. In other words we can directly interpret $\widetilde{\mathcal{A}}_{(1)}^{a}$ as the Kaluza-Klein vectors, $\widetilde{A}_{(1) a}$ as the winding vectors, and $\overrightarrow{\tilde{\phi}}$ as the dilatons coming from the reduction ansatz for the massive IIA theory. In particular, we can see this from the form of the cosmological term in (5.16). From the definitions of $f$ and $\sigma$, we see that the cosmological term is

$$
-\frac{1}{2} m^{2} e^{\frac{5}{2} \phi-\frac{1}{2 \sqrt{7}} \varphi_{1}-\frac{1}{\sqrt{21}} \varphi_{2}}
$$

which is precisely what one obtains by performing a Kaluza-Klein reduction of the cosmological term $-\frac{1}{2} m^{2} e^{\frac{5}{2} \phi}$ in the massive IIA theory. The mass term for the axion $\chi$ in (5.16) can also be understood from this viewpoint, since it is nothing but the Kaluza-Klein reduction of the mass term for the 2 -form $A_{(2)}$ in the ten-dimensional massive IIA theory. 


\section{Conclusions}

In this paper we studied the T-duality maps involving the type IIA and type IIB string theories at the level of the Green-Schwarz sigma-model string actions. This approach provides a string-theoretical derivation of T-dualities, and sheds light on the subsequent web of dualities between the massless and massive type IIA and type IIB string theories.

As a prerequisite for these analyses, we needed explicit (component) forms of the GreenSchwarz actions, in backgrounds including R-R as well as NS-NS massless fields. While the superfield form of the type IIA action in curved backgrounds has been given in [23], the component form, needed for the application of T-duality transformations, was unknown. The component form of the analogous type IIB Green-Schwarz action was also previously unknown. In our derivation of the type IIA Green-Schwarz action, we employed a double dimensional reduction of the eleven-dimensional supermembrane action, and making use of explicit results for the supervielbein and 3-form superpotential, derived up to order $\theta^{2}$ in the fermionic fields [7]. This enabled us to find an explicit and complete form of the type IIA Green-Schwarz action with massless NS-NS and R-R background fields, exact up to order $\theta^{2}$.

This action provided our starting point for studying T-duality transformations at the level of the Green-Schwarz action. We generalised Buscher's T-duality procedure (implemented in [18] in an NSR formalism with only NS-NS background fields) to the GreenSchwarz action, now involving spinor coordinates $\theta$ as well as R-R background fields. Furthermore, we formulated the T-duality transformation rules in terms of adapted backgroundfield parametrizations, thus obtaining more compact and elegant expressions for the Tduality maps which have a natural geometrical interpretation.

These generalised T-duality transformations enabled us in turn to derive the GreenSchwarz action for the type IIB string, giving it for the first time with NS-NS and R-R background fields, exact up to $O\left(\theta^{2}\right)$.

Next, we constructed the string-theoretical T-duality map between the type IIB and massive type IIA strings. Starting with the type IIB Green-Schwarz action, in a background with a $U(1)$ isometry in which the 1 -form field strength $F_{(1)}=d \chi$ is allowed to take a nonvanishing constant value in the direction of the isometry, and applying the generalised T-duality transformation, we derived the Green-Schwarz action for the massive IIA string, in arbitrary R-R and NS-NS maslles backgrounds, again to order $\theta^{2}$. This provides a string theoretical derivation of the T-duality transformation which, at the level of the effective supergravities, corresponds to the equivalence of the Scherk-Schwarz reduced type IIB and 
massive type IIA theories.

In view of the fact that the type IIB string is itself related by T-duality to the massless type IIA string, one can obtain a direct T-duality relation between the massless and massive type IIA strings, by considering them on backgrounds with the two $U(1)$ isometries of a 2-torus. This was discussed by considering D8-branes and D6-branes in the effective field theories in [14]; here, we were able to give a string-theoretic description at the level of T-duality in the Green-Schwarz actions. In addition, we gave a construction of the eightdimensional $S L(3, \mathbb{R}) \times S L(2, \mathbb{R})$ invariant supergravity effective action that includes the two mass parameters $m_{1,2}$, forming a doublet under the $S L(2, \mathbb{R})$ factor. This can be interpreted as a Scherk-Schwarz reduction of the type IIB string on two circles of radii $R_{1}$ and $R_{2}$ (introducing mass parameters $m_{1}$ and $m_{2}$ respectively). The T-duality transformation in turn relates this to the massive type IIA string with one radius compactified on a regular circle and another one Scherk-Schwarz reduced.

Finally, we remark that our discussion of the T-duality mapping between the massless and massive type IIA strings extends straightforwardly, in a manner analogous to that discussed in [14], to a unified picture in which the massive type IIA string emerges in an appropriate limit from the eleven-dimensional supermembrane propagating in a background with the three $U(1)$ isometries of a 3-torus, which can be viewed either as an $S^{1}$ bundle over $T^{2}$ [24] or as a $T^{2}$ bundle over $S^{1}$ 14.

\section{Addendum}

Since writing this paper, and its publication in Nucl. Phys. B573 (2002) 149 and as v1. of hep-th/9907202, we have discovered a small number of minor typographical errors. We have also found that there were various infelicitous choices of convention and notation which, although in no way incorrect, did not contribute positively to the elegance of the results. We are therefore taking the opportunity, while correcting the minor typographical errors, of improving some notation and conventions. We shall set out below what these changes are. For clarity, we shall refer to the versions of this paper published in Nucl. Phys. B573 (2002) 149 and in v1. of hep-th/9907202 as the "old versions," and this current version will be called the "new version."

There were two typographical errors in the old versions that affected our final expressions for the type IIA and type IIB Lagrangians respectively. In the type IIA Lagrangian (2.8), a $\Gamma_{11}$ matrix was accidentally omitted from the $F_{\nu \rho \sigma}$ terms. In the type IIB Lagrangian (3.28), 
the term involving i $\epsilon^{i j}\left(\overline{\tilde{\theta}}^{1} \Gamma_{\mu} D_{j} \tilde{\theta}^{1}-\overline{\tilde{\theta}}^{2} \Gamma_{\mu} D_{j} \tilde{\theta}^{2}\right)$ was accidentally written as $\epsilon^{i j}\left(\overline{\tilde{\theta}}^{1} \Gamma_{\mu} D_{j} \tilde{\theta}^{1}+\right.$ $\left.\overline{\tilde{\theta}}^{2} \Gamma_{\mu} D_{j} \tilde{\theta}^{2}\right)$. Both errors are corrected in this new version.

Turning now to the question of conventions, we found that there were two convention choices in the old versions of this paper that were not helpful to the interpretation of our results.

Firstly, our starting point of the supermembrane action in [0] is written using a convention in which the conjugation of spinors involves the introduction of a factor of i; i.e. the conjugate of a spinor $\psi$ is written as $\bar{\psi}=\mathrm{i} \psi^{\dagger} \Gamma^{t}$, where $\Gamma^{t}$ denotes the Dirac matrix in the time direction. This has the consequence that, for example, the Hermitean Dirac action is written as $\bar{\psi} \Gamma^{\mu} D_{\mu} \psi$ rather than the more familiar i $\bar{\psi} \Gamma^{\mu} D_{\mu} \psi$. To readjust to conventions that are more familiar, we have therefore implemented the replacement of every occurrence of a conjugate spinor by $\mathrm{i}$ times the conjugate spinor in all formula in the old versions of this paper. Thus we have replaced

$$
\bar{\theta}_{\text {old version }} \longrightarrow \mathrm{i} \bar{\theta}_{\text {new version }}
$$

et cetera.

A second inconvenience in our conventions was that when defining the two chiral spinors $\left(\tilde{\theta}^{1}, \tilde{\theta}^{2}\right)$ of the type IIB theory in terms of the spinor $\theta$ of type IIA that has chiral and antichiral projections $\left(\theta^{1}, \theta^{2}\right)$, we defined $\tilde{\theta}^{2}$ as $-\mathrm{i} \Gamma_{0} \theta^{2}$, rather than as $\Gamma_{0} \theta^{2}$ as we are now doing in the this new version of the paper, in equation (3.12). (Recall that $\Gamma_{0}$ is the Dirac matrix in the circle reduction direcion, not the time direction.) Again, there was nothing incorrect about the definition used in the old versions of the paper, but it had the consequence that the two chiral spinors of type IIB were Majorana and anti-Majorana respectively. This led to expressions, such as the old versions of the type IIB Lagrangian (3.28), which although perfectly correct, were somewhat misleading if not set in proper context. In this new version, where we define $\tilde{\theta}^{2}=\Gamma_{0} \theta^{2}$, the two chiral spinors of the type IIB theory are both Majorana. This change of convention amounts to making the replacement of $\tilde{\theta}^{2}$ in the old versions of the paper by $-\mathrm{i} \tilde{\theta}^{2}$, and of $\overline{\tilde{\theta}}^{2}$ by $\mathrm{i} \overline{\tilde{\theta}}^{2}$, in order to get the expressions we are using in this new version.

Finally, we have made a simplification that eliminates the need for certain Dirac-matrix combinations

$$
S_{\mu}{ }^{\nu_{1} \cdots \nu_{p}} \equiv \Gamma_{\mu}^{\nu_{1} \cdots \nu_{p}}-2 p \delta_{\mu}^{\left[\nu_{1}\right.} \Gamma^{\left.\nu_{2} \cdots \nu_{p}\right]}
$$

that we introduced in the old versions of this paper. It is easily seen that these are nothing 
but

$$
S_{\mu}^{\nu_{1} \cdots \nu_{p}}=(-1)^{p} \Gamma^{\nu_{1} \cdots \nu_{p}} \Gamma_{\mu}
$$

and so we have made these replacements in all occurrences in the old versions.

\section{Appendix}

\section{A Massless/massive IIA T-duality from $D=8$ massive su- pergravity}

In this appendix, we discuss the massless/massive IIA T-duality at the level of supergravity. In order to make explicit the T-duality transformation that directly maps between the massless and massive type IIA theories, we shall consider a Scherk-Schwarz dimensional reduction of the massive type IIA supergravity, in which the 2-form field strength $F_{(2)}$ in $D=10$ is allowed to be non-vanishing in the internal directions of the 2-torus. Thus in addition to the mass parameter $m_{1}$ of the original massive IIA theory, we shall also introduce a second mass parameter $m_{2}$ via the Scherk-Schwarz reduction to $D=8$. When $m_{2}=0$, the $D=8$ theory is the standard two-torus reduction of massive type IIA, whilst when $m_{1}=0$, the resuting theory is the Scherk-Schwarz reduction of massless type IIA or M-theory to $D=8$.

Our goal is to show that the $m_{1}=0$ or $m_{2}=0$ cases are in fact the same theory, by giving the explicit transformations of the fields that map one theory to the other. To do so, we shall explicitly exhibit the symmetry of the resulting eight-dimensional theory under the $S L(2, \mathbb{R})$ factor of the $S L(3, \mathbb{R}) \times S L(2, \mathbb{R})$ Cremmer-Julia group, under which $\left(m_{1}, m_{2}\right)$ transform as a doublet. (We can really think of $m_{1}$ and $m_{2}$ as being fields, rather than just parameters; see, for example, [12].) In particular, this $S L(2, \mathbb{R})$ symmetry has a $Z_{2}$ subgroup that maps from the case where $m_{1}=0$ with $m_{2}$ non-vanishing to the case where $m_{2}=0$ with $m_{1}$ non-vanishing. This subgroup, which is the one we found by performing the double-inversion T-duality transformation (5.2) in section 5 , is thus a symmetry that manifestly relates the Scherk-Schwarz reduction of the massless type IIA theory to the ordinary reduction of the massive type IIA theory, in eight dimensions. This then completes the demonstration of the massless/massive type IIA T-duality.

In $D=10$, the massive type IIA theory [9] is given by

$$
\begin{aligned}
\mathcal{L}= & R * \mathbb{1}-\frac{1}{2} * d \phi \wedge d \phi-\frac{1}{2} e^{\frac{3}{2} \phi} * \hat{F}_{(2)} \wedge \hat{F}_{(2)}-\frac{1}{2} e^{-\phi} * \hat{F}_{(3)} \wedge F_{(3)}-\frac{1}{2} e^{\frac{1}{2} \phi} * \hat{F}_{(4)} \wedge F_{(4)} \\
& -\frac{1}{2} d \hat{A}_{(3)} \wedge d \hat{A}_{(3)} \wedge \hat{A}_{(2)}-\frac{1}{6} m_{1} d \hat{A}_{(3)} \wedge\left(\hat{A}_{(2)}\right)^{3}-\frac{1}{40} m_{1}^{2}\left(\hat{A}_{(2)}\right)^{5}-\frac{1}{2} m_{1}^{2} e^{\frac{5}{2} \phi} * \mathbb{1},(\mathrm{A} .1)
\end{aligned}
$$


where the field strengths are given in terms of potentials by

$$
\begin{aligned}
& \hat{F}_{(2)}=d \hat{A}_{(1)}+m_{1} \hat{A}_{(2)}, \quad \hat{F}_{(3)}=d \hat{A}_{(2)} \\
& \hat{F}_{(4)}=d \hat{A}_{(3)}+\hat{A}_{(1)} \wedge d \hat{A}_{(2)}+\frac{1}{2} m_{1} \hat{A}_{(2)} \wedge \hat{A}_{(2)} .
\end{aligned}
$$

We now reduce on $T^{2}$ the standard way, using the conventions and notation of [19], adapted to the case where the first reduction step is from $D=10$ to $D=9$, and where the generalised Scherk-Schwarz reduction is included. To be explicit, we have

$$
\begin{aligned}
& \hat{A}_{(1)}=A_{(1)}+A_{(0) \alpha} d z^{\alpha}-m_{2} z^{2} d z^{1} \\
& \hat{A}_{(2)}=A_{(2)}+A_{(1) \alpha} \wedge d z^{\alpha}+A_{(0) 12} d z^{1} \wedge d z^{2}, \\
& \hat{A}_{(4)}=A_{(3)}+A_{(2) \alpha} \wedge d z^{\alpha}+A_{(1) 12} \wedge d z^{1} \wedge d z^{2}+\hat{A}_{1} \wedge \hat{A}_{(2)} .
\end{aligned}
$$

Note that the last term in $\hat{A}_{(4)}$, which amounts to a field redefinition, is needed in order that the coordinate $z^{2}$ does not appear undifferentiated in the Lagrangian. The ansatz for the Einstein-frame metric is $e^{\phi / 2} d \hat{s}_{10}^{2}$ where $d \hat{s}_{10}^{2}$ is the string frame metric given in (5.3).

We find that the Scherk-Schwarz reduced Lagrangian in $D=8$ is given by

$$
\begin{aligned}
\mathcal{L}_{8}= & R * \mathbb{1}-\frac{1}{2} * d f \wedge d f-\frac{1}{2} e^{2 f} * d \chi \wedge d \chi-\frac{1}{2} * d \psi \wedge d \psi-\frac{1}{2} e^{2 \psi} * d \mathcal{A}_{(0) 2}^{1} \wedge d \mathcal{A}_{(0) 2}^{1} \\
& -\frac{1}{2} * d \sigma \wedge d \sigma-\frac{1}{2} e^{-\psi+\sqrt{3} \sigma} * F_{(1) 1} \wedge F_{(1) 1}-\frac{1}{2} e^{\psi+\sqrt{3} \sigma} * F_{(1) 2} \wedge F_{(1) 2} \\
& -\frac{1}{2} e^{-f} * F_{(4)} \wedge F_{(4)}-\frac{1}{2} e^{-\frac{2}{\sqrt{3}} \sigma} * F_{(3)} \wedge F_{(3)} \\
& -\frac{1}{2} e^{\frac{1}{\sqrt{3}} \sigma}\left(e^{-\psi} * F_{(3) 1} \wedge F_{(3) 1}+e^{\psi} * F_{(3) 2} \wedge F_{(3) 2}\right) \\
& -\frac{1}{2} e^{\frac{2}{\sqrt{3}} \sigma}\left(e^{-f} * F_{(2)} \wedge F_{(2)}+e^{f} * F_{(2) 12} \wedge F_{(2) 12}\right) \\
& -\frac{1}{2} e^{-\psi-\frac{1}{\sqrt{3}} \sigma}\left(e^{-f} * \mathcal{F}_{(2)}^{2} \wedge \mathcal{F}_{(2)}^{2}+e^{f} * F_{(2) 1} \wedge F_{(2) 1}\right) \\
& -\frac{1}{2} e^{\psi-\frac{1}{\sqrt{3}} \sigma}\left(e^{-f} * \mathcal{F}_{(2)}^{1} \wedge \mathcal{F}_{(2)}^{1}+e^{f} * F_{(2) 2} \wedge F_{(2) 2}\right) \\
& -\frac{1}{2} e^{\frac{4}{\sqrt{3}} \sigma}\left(m_{1}^{2} e^{-f}+\left(m_{2}+m_{1} \chi\right)^{2} e^{f}\right) * \mathbb{1}+\mathcal{L}_{F F A}
\end{aligned}
$$

where $\chi=A_{(0) 12}$ and

$$
\mathcal{L}_{F F A}=-\frac{1}{2} \chi F_{(4)} \wedge F_{(4)}+\Omega_{1} \wedge d A_{(3)}+\Omega_{2},
$$

with

$$
\begin{aligned}
\Omega_{1}= & \epsilon^{\alpha \beta} A_{(1) \alpha} d A_{(2) \beta}+\frac{1}{2} \epsilon^{\alpha \beta} A_{(1)} A_{(1) \alpha} d A_{(1) \beta}-A_{(2)} d A_{(1) 12} \\
& +\frac{1}{2} A_{(1) 12} A_{(1) \alpha} d \mathcal{A}_{(1)}^{\alpha}+A_{(1) 1} A_{(1) 2} d A_{(1)}+m_{1} A_{(1) 1} A_{(1) 2} A_{(2)} \\
& -\frac{1}{2} m_{2}\left(A_{(2)}\right)^{2}-\frac{1}{2} m_{2} A_{(2)} A_{(1) \alpha} \mathcal{A}_{(1)}^{\alpha}+\frac{1}{4} m_{2} A_{(1) 1} A_{(1) 2} \mathcal{A}_{(1)}^{1} \mathcal{A}_{(1)}^{2} .
\end{aligned}
$$


The expression for $\Omega_{2}$, which does not involve $A_{(3)}$, is quite complicated, and we shall not present it here. The exterior derivative of $\mathcal{L}_{F F A}$ is, however, rather simple, and is given by

$$
d \mathcal{L}_{F F A}=-\frac{1}{2} d\left(\chi F_{(4)} \wedge F_{(4)}\right)+d \Omega_{1} \wedge F_{(4)}+F_{(3)} \wedge F_{(3) 1} \wedge F_{(3) 2}
$$

In the scalar sector, the $S L(2, \mathbb{R})$ symmetry is generated by the axion $\chi \equiv A_{(0) 12}$, and the dilaton combination $f$, given by (5.4), which arises in the exponential prefactor for the axion's kinetic term in $D=8$. To make the $S L(2, \mathbb{R})$ manifest, it is useful to perform a number of redefinitions of the dimensionally-reduced potentials. These are given below, in equation (A.17). Expressed in terms of the now-redefined potentials, we obtain the following expressions for the various field strengths in $D=8$. Firstly, we have

$$
F_{(3)}=d A_{(2)}-\frac{1}{2} A_{(1) \alpha} d \mathcal{A}_{(1)}^{\alpha}-\frac{1}{2} \mathcal{A}_{(1)}^{\alpha} d A_{(1) \alpha},
$$

which is a singlet under the $S L(2, \mathbb{R})$. We find that the remaining two 3-form field strengths are also $S L(2, \mathbb{R})$ singlets, and are given by

$$
\begin{aligned}
F_{(3) 1}= & d A_{(2) 1}+\frac{1}{2} A_{(1)} d A_{(1) 1}+\frac{1}{2} A_{(1) 1} d A_{(1)}+\frac{1}{2} A_{(1) 12} d \mathcal{A}_{(1)}^{2}+\frac{1}{2} \mathcal{A}_{(1)}^{2} d A_{(1) 12}-A_{(0) 1} F_{(3)} \\
& +A_{(2)}\left(m_{1} A_{(1) 1}+m_{2} \mathcal{A}_{(1)}^{2}\right)+\frac{1}{2} A_{(1) 1} \mathcal{A}_{(1)}^{2}\left(m_{1} A_{(1) 2}-m_{2} \mathcal{A}_{(1)}^{1}\right) \\
F_{(3) 2}= & d A_{(2) 2}+\frac{1}{2} A_{(1)} d A_{(1) 2}+\frac{1}{2} A_{(1) 2} d A_{(1)}-\frac{1}{2} A_{(1) 12} d \mathcal{A}_{(1)}^{1}-\frac{1}{2} \mathcal{A}_{(1)}^{1} d A_{(1) 12}-A_{(0) 2} F_{(3)} \\
& -\mathcal{A}_{(0) 2}^{1} F_{(3) 1}+A_{(2)}\left(m_{1} A_{(1) 2}-m_{2} \mathcal{A}_{(1)}^{1}\right)+\frac{1}{2} A_{(1) 2} \mathcal{A}_{(1)}^{1}\left(m_{1} A_{(1) 1}+m_{2} \mathcal{A}_{(1)}^{2}\right) .
\end{aligned}
$$

We also have the pair of singlet 1-forms,

$$
\begin{aligned}
& F_{(1) 1}=d A_{(0) 1}+\left(m_{1} A_{(1) 1}+m_{2} \mathcal{A}_{(1)}^{2}\right) \\
& F_{(1) 2}=d A_{(0) 2}-\mathcal{A}_{(0) 2}^{1} F_{(1) 1}+\left(m_{1} A_{(1) 2}-m_{2} \mathcal{A}_{(1)}^{1}\right) .
\end{aligned}
$$

Next, we find

$$
\begin{aligned}
F_{(2)}= & d A_{(1)}+A_{(0) \alpha} d \mathcal{A}_{(1)}^{\alpha}+m_{1} A_{(2)}-\frac{1}{2} m_{1} A_{(1) \alpha} \mathcal{A}_{(1)}^{\alpha}+m_{2} \mathcal{A}_{(1)}^{1} \mathcal{A}_{(1)}^{2}, \\
F_{(2) 12}= & d A_{(1) 12}+A_{(0) 1} d A_{(1) 2}-A_{(0) 2} d A_{(1) 1}+\chi F_{(2)} \\
& +m_{2} A_{(2)}+\frac{1}{2} m_{2} A_{(1)} \mathcal{A}_{(1)}^{\alpha}-m_{1} A_{(1) 1} A_{(1) 2}
\end{aligned}
$$

This pair of field strengths forms a doublet under the $S L(2, \mathbb{R})$ we are considering. For the other 2-forms, we find that they form two pairs, namely

$$
\begin{aligned}
\mathcal{F}_{(2)}^{2} & =d \mathcal{A}_{(1)}^{2}, \\
F_{(2) 1} & =d A_{(1) 1}-\chi d \mathcal{A}_{(1)}^{2},
\end{aligned}
$$


and

$$
\begin{aligned}
\mathcal{F}_{(2)}^{1} & =d \mathcal{A}_{(1)}^{1}+\mathcal{A}_{(0) 2}^{1} d \mathcal{A}_{(1)}^{2}, \\
F_{(2) 2} & =d A_{(1) 2}+\chi d \mathcal{A}_{(1)}^{1}-\mathcal{A}_{(0) 2}^{1} F_{(2) 1}
\end{aligned}
$$

Each pair is a doublet under the $S L(2, \mathbb{R})$.

Finally, we have the 4 -form. This turns out to be

$$
\begin{aligned}
F_{(4)}= & d A_{(3)}+A_{(1)} d A_{(2)}+\mathcal{A}_{(1)}^{\alpha} d A_{(2) \alpha}-\frac{1}{2} A_{(1)} \mathcal{A}_{(1)}^{\alpha} d A_{(1) \alpha} \\
& -\frac{1}{2} A_{(1) 12}\left(\mathcal{A}_{(1)}^{1} d A_{(1) 2}-\mathcal{A}_{(1)}^{2} d A_{(1) 1}\right)+\frac{1}{2} m_{1}\left(A_{(2)}\right)^{2}-\frac{1}{2} m_{1} A_{(2)} A_{(1) \alpha} \mathcal{A}_{(1)}^{\alpha} \\
& -\frac{1}{4} m_{1} A_{(1) 1} A_{(1) 2} \mathcal{A}_{(1)}^{1} \mathcal{A}_{(1)}^{2}+m_{2} A_{(2)} \mathcal{A}_{(1)}^{1} \mathcal{A}_{(1)}^{2} .
\end{aligned}
$$

This, together with its Hodge dual, forms a doublet under the $S L(2, \mathbb{R})$. The symmetry involving $F_{(4)}$ is therefore seen only at the level of the field equations.

It is useful at this stage to consider the various doublet symmetries under the $S L(2, \mathbb{R})$ generated by $f$ and $\chi$ in more detail. First of all, we note from (A.4) that, as one would expect, $f$ does not couple to the kinetic terms for any of the $S L(2, \mathbb{R})$-singlet fields. All the doublet pairs of 2 -form fields strengths $\left(F_{+}, F_{-}\right)$, with potentials $\left(A_{+}, A_{-}\right)=\left(A_{(1)}, A_{(1) 12}\right)$, $\left(-\mathcal{A}_{(1)}^{2}, A_{(1) 1}\right)$ and $\left(\mathcal{A}_{(1)}^{1}, A_{(1) 2}\right)$, which we exhibited above, have kinetic terms which couple to $f$ in the form $-\frac{1}{2} e^{-f} F_{+}^{2}-\frac{1}{2} e^{f}\left(F_{-}+\chi F_{+}\right)^{2}$. Under $S L(2, \mathbb{R})$ transformations acting on $\tau=\chi+\mathrm{i} e^{-f}$ via the fractional linear mapping $\tau \longrightarrow(a \tau+b) /(c \tau+d)$, the potentials $\left(A_{+}, A_{-}\right)$transform as

$$
\left(\begin{array}{c}
A_{+} \\
A_{-}
\end{array}\right) \longrightarrow\left(\begin{array}{cc}
d & -c \\
-b & a
\end{array}\right)\left(\begin{array}{l}
A_{+} \\
A_{-}
\end{array}\right) .
$$

The two mass parameters $\left(m_{1}, m_{2}\right)$ transform in the same way as $\left(A_{+}, A_{-}\right)$. Finally, to discuss the $S L(2, \mathbb{R})$ symmetry for $F_{(4)}$, we note that the pair $F_{(4)}$ and $\left(e^{-f} * F_{(4)}+\chi F_{(4)}\right)$ transform like the upper and lower components of a doublet, as in (A.15). Since this involves the 4 -form and its dual, the $S L(2, \mathbb{R})$ symmetry is realised here at the level of the equations of motion rather than the Lagrangian. The the Bianchi identity and the equation of motion for the 4-form are given by

$$
d F_{(4)}=X, \quad d\left(e^{-f} * F_{(4)}+\chi F_{(4)}\right)=d \Omega_{1},
$$

where $X$ can be easily read off from (A.14). It is straightforward to verify that these two equations form a doublet under the $S L(2, \mathbb{R})$.

For completeness, we list the field redefinitions that we made for the potentials. Making the following substitutions in the expressions for the field strengths directly following from 
the dimensional reduction, we obtain the field strengths given above:

$$
\begin{aligned}
& A_{(3)} \longrightarrow A_{(3)}-A_{(2)} A_{(1)},-A_{(2)} A_{(0) \alpha} \mathcal{A}_{(1)}^{\alpha}+\frac{1}{2} \epsilon^{\alpha \beta} A_{(0) \alpha} A_{(1) \beta} \mathcal{A}_{(1)}^{1} \mathcal{A}_{(1)}^{2}, \\
& A_{(2)} \quad \longrightarrow \quad A_{(2)}+\frac{1}{2} A_{(1) \alpha} \mathcal{A}_{(1)}^{\alpha}+\chi \mathcal{A}_{(1)}^{1} \mathcal{A}_{(1)}^{2} \text {, } \\
& A_{(1)} \longrightarrow A_{(1)}+A_{(0) \alpha} \mathcal{A}_{(1) \alpha}, \\
& A_{(2) 1} \quad \longrightarrow \quad A_{(2) 1}-A_{(0) 1} A_{(2)}-\frac{1}{2} A_{(1)} A_{(1) 1}-\frac{1}{2} A_{(1) 12} \mathcal{A}_{(1)}^{2} \\
& +\frac{1}{2} A_{(0) 1}\left(A_{(1) 1} \mathcal{A}_{(1)}^{1}-A_{(1) 2} \mathcal{A}_{(1)}^{2}\right)+A_{(0) 2} A_{(1) 1} \mathcal{A}_{(1)}^{2}, \\
& A_{(2) 2} \quad \longrightarrow \quad A_{(2) 2}-A_{(0) 2} A_{(2)}-\frac{1}{2} A_{(1)} A_{(1) 2}+\frac{1}{2} A_{(1) 12} \mathcal{A}_{(1)}^{1} \\
& -\frac{1}{2} A_{(0) 2}\left(A_{(1) 1} \mathcal{A}_{(1)}^{1}-A_{(1) 2} \mathcal{A}_{(1)}^{2}\right)+A_{(0) 1} A_{(1) 2} \mathcal{A}_{(1)}^{1}, \\
& A_{(1) 1} \quad \longrightarrow \quad A_{(1) 1}-\chi \mathcal{A}_{(1)}^{2} \text {, } \\
& A_{(1) 2} \longrightarrow A_{(1) 2}+\chi \mathcal{A}_{(1)}^{1}, \\
& A_{(1) 12} \longrightarrow A_{(1) 12}+\epsilon^{\alpha \beta} A_{(0) \alpha} A_{(1) \beta}, \\
& \mathcal{A}_{(1)}^{1} \longrightarrow \mathcal{A}_{(1)}^{1}+\mathcal{A}_{(0) 2}^{1} \mathcal{A}_{(1)}^{2} \text {. }
\end{aligned}
$$

To summarise the appendix, we have constructed a manifestly $S L(3, \mathbb{R}) \times S L(2, \mathbb{R})$ invariant massive supergravity in $D=8$, with two mass parameters $m_{1}$ and $m_{2}$ which form a doublet under the $S L(2, \mathbb{R})$ factor. When $m_{2}=0$ with $m_{1}$ non-vanishing, the theory is the standard Kaluza-Klein 2-torus reduction of the massive type IIA theory in $D=10$. On the other hand when $m_{1}=0$ with $m_{2}$ non-vanishing, the theory comes from the the Scherk-Schwarz reduction of massless type IIA (or M-theory). The manifest $S L(2, \mathbb{R}$ ) symmetry implies in particular that these two eight-dimensional cases are equivalent. In particular, as we observed in section 5 , the discrete transformation that maps $\left(m_{1}, 0\right)$ to $\left(0, m_{2}\right)$, associated with the massive/massless type IIA theories, interchanges $R_{1} \leftrightarrow 1 / R_{2}$. This $\mathbb{Z}_{2}$ is a subgroup of the $S L(2, \mathbb{R})$; it is quite different from the $R_{1} \leftrightarrow 1 / R_{1} \mathbb{Z}_{2}$ transformation that relates the type IIA and type IIB theories, which is intrinsically discrete and is not part of any connected group.

As was observed in [24], the U-duality group can be decomposed as general coordinate transformations of the internal spaces of either the type IIA or the type IIB theories, together with the T-duality transformation that maps between the two theories. From the type IIB point of view, the $S L(2, \mathbb{R})$ symmetry under which $\left(m_{1}, m_{2}\right)$ form a doublet is nothing but a residual general coordinate transformation symmetry of the internal 2-torus. This is because in the type IIB case, the axion $\chi$ in $D=10$ can be Scherk-Schwarz reduced on each of the two circles reductions, giving rise to two mass parameters that naturally form a doublet under the $S L(2, \mathbb{R})$ residuum of the 2-torus general coordinate transformations. 


\section{Note added}

After this work was completed, a paper appeared which also considers certain aspects of type IIA/IIB T-duality for R-R fields [25].

\section{Acknowledgements}

We are grateful to Chris Hull, Igor Lavrinenko and Arkady Tseytlin for useful discussions, and to SISSA for hospitality.

\section{References}

[1] E. Alvarez, L. Alvarez-Gaume and Y. Lozano, An Introduction to T duality in string theory, Nucl. Phys. Proc. Suppl. 41 (1995) 1, hep-th/9410237.

[2] A. Giveon, M. Porrati and E. Rabinovici, Target space duality in string theory, Phys. Rept. 244 (1994) 77, hep-th/9401139.

[3] M.J. Duff, P.S. Howe, T. Inami and K.S. Stelle, Superstrings in $D=10$ from supermembranes in $D=11$, Phys. Lett. 191B (1987) 70.

[4] E. Bergshoeff, E. Sezgin and P.K. Townsend, Supermembranes and eleven-dimensional supergravity, Phys. Lett. B189 (1987) 75.

[5] E. Cremmer and S. Ferrara, Formulation of eleven-dimensional supergravity in superspace, Phys. Lett. B91 (1980) 61.

[6] L. Brink and P. Howe, Eleven-dimensional supergravity on the mass-shell in superspace, Phys. Lett. B91 (1980) 384.

[7] B. de Wit, K. Peeters and J. Plefka, Superspace geometry for supermembrane backgrounds, Nucl. Phys. B532 (1998) 99, hep-th/9803209.

[8] E. Bergshoeff, C.M. Hull and T. Ortin, Duality in the type II superstring effective action, Nucl. Phys. B451 (1995) 547, hep-th/9504081.

[9] L.J. Romans, Massive $N=2$ a supergravity in ten dimensions, Phys. Lett. B169 (1986) 374. 
[10] E. Bergshoeff, M. de Roo, M.B. Green, G. Papadopoulos and P.K. Townsend, Duality of type II 7 branes and 8 branes, Nucl. Phys. B470 (1996) 113, hep-th/9601150.

[11] P. Meessen and T. Ortin, An Sl(2,Z) Multiplet of Nine-Dimensional Type II Supergravity Theories, Nucl. Phys. B541 (1999) 195, hep-th/9806120.

[12] I.V. Lavrinenko, H. Lü, C.N. Pope and K.S. Stelle, Superdualities, brane tensions and massive IIA / IIB duality, Nucl. Phys. B555 (1999) 201, hep-th/9903057.

[13] P.M. Cowdall, H. Lü, C.N. Pope, K.S. Stelle and P.K. Townsend, Domain walls in massive supergravities, Nucl. Phys. B486 (1997) 49, hep-th/9608173.

[14] C.M. Hull, Massive string theories from $M$ theory and F theory, JHEP 11 (1998) 027, hep-th/9811021.

[15] A. Achucarro, P. Kapusta and K.S. Stelle, Strings from membranes: The origin of conformal invariance, Phys. Lett. B232 (1989) 302.

[16] M.T. Grisaru, H. Nishino and D. Zanon, Beta functions for the Green-Schwarz superstring, Nucl. Phys. B314 (1989) 363.

[17] A.A. Tseytlin, On dilaton dependence of type II superstring action, Class. Quant. Grav. 13 (1996) L81, hep-th/9601109.

[18] T.H. Buscher, A symmetry of the string background field equations, Phys. Lett. B194 (1987) 59 .

[19] E. Cremmer, B. Julia, H. Lü and C.N. Pope, Dualisation of dualities, Nucl. Phys. B523 (1998) 73, hep-th/9710119.

[20] I.V. Lavrinenko, H. Lü and C.N. Pope, Fibre bundles and generalised dimensional reduction, Class. Quant. Grav. 15 (1998) 2239, hep-th/9710243.

[21] M. Fukuma, T. Oota and H. Tanaka, Comments on T dualities of Ramond-Ramond potentials on tori, hep-th/9907132.

[22] A. Giveon and M. Roček, Generalized duality in curved string backgrounds, Nucl. Phys. B380 (1992) 128, hep-th/9112070.

[23] M.T. Grisaru, P. Howe, L. Mezincescu, B. Nilsson and P.K. Townsend, $N=2$ superstrings in a supergravity background, Phys. Lett. B162 (1985) 116. 
[24] I.V. Lavrinenko, H. Lü, C.N. Pope and T.A. Tran, U-duality as general coordinate transformations, and spacetime geometry, Int. J. Mod. Phys. A14 (1999) 4915, hepth/9807006.

[25] S.F. Hassan, T-duality, space-time spinors and R-R fields in curved backgrounds, hepth/9907152. 